\title{
ECOC comparison exercise with identical thermal protocols after temperature offsets correction - instrument diagnostics by in-depth evaluation of operational parameters
}

\author{
P. Panteliadis et al.
}

Correspondence to: P. Panteliadis (ppanteliadis@ggd.amsterdam.nl) 
Supplemental material: ECOC comparison exercise with identical thermal protocols after temperature offsets correction. Instrument diagnostics by in-depth evaluation of operational parameters.

Table S1. Normalized mean $(\mathrm{m})$, repeatability $\left(\mathrm{s}_{\mathrm{r}}\right)$ and reproducibility $\left(\mathrm{sL}_{\mathrm{L}}\right)$ relative standard deviation, raw and corrected consensus values for pooled TC results, EUSAAR2 and NIOSH870, per filter.

\begin{tabular}{|c|c|c|c|c|c|}
\hline Sample & $\begin{array}{l}\text { Consensus } \\
\text { values }\end{array}$ & $\mathbf{m}$ & $\mathbf{S r}$ & SR & $\begin{array}{c}\text { Participan } \\
\text { ts }\end{array}$ \\
\hline \multirow{2}{*}{ A } & Raw & 21.90 & $\begin{array}{c}3.31 \\
(15.1 \%)\end{array}$ & $\begin{array}{c}4.15 \\
(19.0 \%)\end{array}$ & 17 \\
\hline & Corrected & 21.20 & $\begin{array}{c}2.35 \\
(11.1 \%)\end{array}$ & $\begin{array}{c}2.96 \\
(14.0 \%)\end{array}$ & 15 \\
\hline \multirow{2}{*}{ B } & Raw & 79.02 & $\begin{array}{c}12.06 \\
(15.3 \%)\end{array}$ & $\begin{array}{c}15.02 \\
(19.0 \%)\end{array}$ & 16 \\
\hline & Corrected & 77.03 & $\begin{array}{c}6.58 \\
(8.5 \%)\end{array}$ & $\begin{array}{c}8.22 \\
(10.7 \%)\end{array}$ & 13 \\
\hline \multirow{2}{*}{ D } & Raw & 10.09 & $\begin{array}{c}1.42 \\
(14.1 \%)\end{array}$ & $\begin{array}{c}1.76 \\
(17.5 \%)\end{array}$ & 16 \\
\hline & Corrected & 9.74 & $\begin{array}{c}1.14 \\
(11.7 \%)\end{array}$ & $\begin{array}{c}1.41 \\
(14.5 \%)\end{array}$ & 14 \\
\hline \multirow{2}{*}{ G } & Raw & 18.71 & $\begin{array}{c}2.73 \\
(14.6 \%)\end{array}$ & $\begin{array}{c}3.42 \\
(18.3 \%)\end{array}$ & 17 \\
\hline & Corrected & 18.28 & $\begin{array}{c}2.12 \\
(11.6 \%)\end{array}$ & $\begin{array}{c}2.67 \\
(14.6 \%)\end{array}$ & 16 \\
\hline \multirow{2}{*}{$\mathbf{U}$} & Raw & 35.55 & $\begin{array}{c}5.71 \\
(16.1 \%)\end{array}$ & $\begin{array}{c}7.09 \\
(19.9 \%)\end{array}$ & 16 \\
\hline & Corrected & 34.28 & $\begin{array}{c}3.73 \\
(10.9 \%)\end{array}$ & $\begin{array}{c}4.64 \\
(13.5 \%)\end{array}$ & 14 \\
\hline
\end{tabular}


Table S2. TC results per participant and protocol in $\mu \mathrm{g} / 10 \mu \mathrm{L}$ for the sucrose solutions (S1, S2) and in $\mu \mathrm{g} / \mathrm{cm}^{2}$ for the filter samples.

\begin{tabular}{|c|c|c|c|c|c|c|c|c|}
\hline Laboratory & TC & S1 & S2 & $\mathbf{A}$ & B & D & G & $\mathbf{U}$ \\
\hline \multirow{6}{*}{1} & \multirow{3}{*}{ EUSAAR2 } & 9.92 & 32.05 & 20.75 & 73.56 & 8.49 & 17.32 & 33.92 \\
\hline & & 10.17 & 32.51 & 20.44 & 73.65 & 8.10 & 16.66 & 31.97 \\
\hline & & 9.77 & 32.08 & & & & & \\
\hline & \multirow{3}{*}{ NIOSH870 } & 10.24 & 33.27 & 20.51 & 73.55 & 9.65 & 17.45 & 31.44 \\
\hline & & 10.01 & 33.35 & 20.90 & 72.49 & 9.11 & 17.37 & 32.28 \\
\hline & & 9.82 & 33.52 & & & & & \\
\hline \multirow{6}{*}{2} & \multirow{3}{*}{ EUSAAR2 } & 10.09 & 34.89 & 20.58 & 76.61 & 9.16 & 17.60 & 35.57 \\
\hline & & 10.71 & 35.28 & 20.67 & 80.21 & 9.07 & 17.16 & 33.38 \\
\hline & & 10.05 & 35.30 & & & & & \\
\hline & \multirow{3}{*}{ NIOSH870 } & 10.16 & 35.32 & 21.46 & 75.04 & 10.57 & 18.44 & 35.93 \\
\hline & & 10.52 & 35.24 & 21.23 & 72.66 & 9.44 & 18.32 & 32.48 \\
\hline & & 10.78 & 34.46 & & & & & \\
\hline \multirow{6}{*}{3} & \multirow{3}{*}{ EUSAAR2 } & 10.24 & 34.65 & 26.04 & 78.61 & 10.20 & 17.84 & 33.31 \\
\hline & & 10.61 & 32.50 & 21.11 & 73.40 & 9.18 & 17.95 & 30.41 \\
\hline & & 9.86 & 33.88 & & & & & \\
\hline & \multirow{3}{*}{ NIOSH930 } & 10.80 & 35.44 & 21.54 & 73.69 & 9.91 & 18.75 & 30.86 \\
\hline & & 10.18 & 33.69 & 22.51 & 76.81 & 9.45 & 18.50 & 29.42 \\
\hline & & 10.84 & 35.25 & & & & & \\
\hline \multirow{6}{*}{4} & \multirow{3}{*}{ EUSAAR2 } & 10.97 & 34.30 & 18.72 & 68.92 & 9.26 & 17.66 & 30.92 \\
\hline & & 9.19 & 33.95 & 18.39 & 74.05 & 9.87 & 17.55 & 32.59 \\
\hline & & 9.06 & 33.54 & & & & & \\
\hline & \multirow{3}{*}{ NIOSH870 } & 10.60 & 32.74 & 18.71 & 68.70 & 9.92 & 17.33 & 31.89 \\
\hline & & 10.85 & 32.07 & 18.47 & 67.26 & 9.23 & 17.29 & 29.80 \\
\hline & & 10.62 & 32.83 & & & & & \\
\hline \multirow{6}{*}{5} & \multirow{3}{*}{ EUSAAR2 } & 9.90 & 32.30 & 19.25 & 77.48 & 9.11 & 16.49 & 32.13 \\
\hline & & 9.80 & 32.10 & 20.76 & 73.04 & & 16.76 & 32.01 \\
\hline & & 9.70 & 32.70 & & & & & \\
\hline & \multirow{3}{*}{ NIOSH-like } & 10.00 & 32.40 & 20.17 & 74.98 & 9.69 & 17.54 & 34.48 \\
\hline & & 10.10 & 33.10 & 20.50 & 72.52 & 9.83 & 17.46 & 33.62 \\
\hline & & 10.10 & 33.00 & & & & & \\
\hline
\end{tabular}


Table S2 (continued). TC results per participant and protocol in $\mu \mathrm{g} / 10 \mu \mathrm{L}$ for the sucrose solutions $(\mathrm{S1}, \mathrm{S2})$ and in $\mu \mathrm{g} / \mathrm{cm}^{2}$ for the filter samples.

\begin{tabular}{|c|c|c|c|c|c|c|c|c|}
\hline Laboratory & TC & S1 & S2 & $\mathbf{A}$ & B & D & G & $\mathbf{U}$ \\
\hline \multirow{6}{*}{6} & \multirow{3}{*}{ EUSAAR2 } & 9.73 & 32.85 & 21.35 & 86.94 & 10.05 & 17.43 & 37.61 \\
\hline & & 9.72 & 33.13 & 21.67 & 79.87 & 9.54 & 17.55 & 36.15 \\
\hline & & 9.68 & 33.14 & & & & & \\
\hline & \multirow{3}{*}{ NIOSH870 } & 10.03 & 33.33 & 21.35 & 72.87 & 9.27 & 18.10 & 34.80 \\
\hline & & 9.97 & 33.49 & 21.16 & 77.42 & 9.29 & 17.75 & 33.22 \\
\hline & & 9.97 & 33.49 & & & & & \\
\hline \multirow{8}{*}{7} & \multirow{4}{*}{ EUSAAR2 } & 9.88 & 33.18 & 21.25 & & & 17.80 & \\
\hline & & 9.86 & 33.23 & 18.96 & & & 16.94 & \\
\hline & & 10.21 & 33.08 & 18.65 & & & 16.63 & \\
\hline & & & & 18.84 & & & 17.48 & \\
\hline & \multirow{4}{*}{ NIOSH870 } & 10.30 & 32.95 & 20.18 & 73.15 & 9.02 & 16.87 & 30.85 \\
\hline & & 10.45 & 33.45 & 20.58 & 74.77 & 9.27 & 17.30 & 32.85 \\
\hline & & 10.37 & 33.89 & 18.91 & 70.30 & 9.50 & 17.20 & 32.74 \\
\hline & & & & 20.38 & 71.86 & 9.17 & 17.33 & 33.12 \\
\hline \multirow{6}{*}{8} & \multirow{3}{*}{ EUSAAR2 } & 9.94 & 32.82 & 20.05 & 76.30 & 9.51 & 17.89 & 36.12 \\
\hline & & 10.07 & 33.05 & 20.07 & 78.07 & 9.24 & 17.42 & 34.31 \\
\hline & & 10.85 & 33.80 & & & & & \\
\hline & \multirow{3}{*}{ NIOSH870 } & 9.94 & 34.57 & 20.22 & 74.62 & 9.56 & 17.88 & 34.48 \\
\hline & & 10.39 & 34.25 & 20.27 & 78.86 & 9.50 & 18.63 & 33.86 \\
\hline & & 10.50 & 34.36 & & & & & \\
\hline \multirow{6}{*}{9} & \multirow{3}{*}{ EUSAAR2 } & 10.44 & 32.75 & 21.09 & 75.57 & 11.79 & 16.52 & 38.94 \\
\hline & & 11.11 & 34.93 & 21.06 & 76.55 & 9.49 & 18.60 & 34.90 \\
\hline & & 10.78 & 34.88 & & & & & \\
\hline & \multirow{3}{*}{ NIOSH870 } & 10.87 & 31.69 & 20.52 & 68.59 & 9.04 & 16.75 & 30.67 \\
\hline & & 9.96 & 33.71 & 20.14 & 69.47 & 9.97 & 17.59 & 30.83 \\
\hline & & 10.29 & 32.56 & & & & & \\
\hline \multirow{6}{*}{10} & \multirow{3}{*}{ EUSAAR2 } & 14.04 & 43.32 & 26.41 & 94.79 & 9.84 & 23.77 & 42.50 \\
\hline & & 12.64 & 41.91 & 26.13 & 93.23 & 11.14 & 24.58 & 40.48 \\
\hline & & 12.39 & 42.92 & & & & & \\
\hline & \multirow{3}{*}{ NIOSH-like } & 12.47 & 37.61 & 30.41 & 93.36 & 15.48 & 25.21 & 47.20 \\
\hline & & 12.28 & 44.49 & 27.26 & 95.67 & 12.26 & 25.13 & 46.77 \\
\hline & & 9.71 & 36.57 & & & & & \\
\hline \multirow{6}{*}{11} & \multirow{3}{*}{ EUSAAR2 } & 16.21 & 52.72 & 29.75 & 105.90 & 12.18 & 24.89 & 51.96 \\
\hline & & 15.83 & 51.50 & 29.68 & 104.92 & 11.81 & 23.95 & 51.98 \\
\hline & & 17.80 & 53.93 & & & & & \\
\hline & \multirow{3}{*}{ NIOSH870 } & 17.71 & 54.55 & 30.75 & 114.58 & 13.81 & 27.99 & 46.58 \\
\hline & & 17.65 & 56.51 & 30.82 & 110.04 & 13.69 & & 47.83 \\
\hline & & 18.03 & 53.29 & & & & & \\
\hline
\end{tabular}


Table S2 (continued). TC results per participant and protocol in $\mu \mathrm{g} / 10 \mu \mathrm{L}$ for the sucrose solutions (S1, S2) and in $\mu \mathrm{g} / \mathrm{cm}^{2}$ for the filter samples.

\begin{tabular}{|c|c|c|c|c|c|c|c|c|}
\hline Laboratory & TC & S1 & S2 & A & B & D & G & $\mathbf{U}$ \\
\hline \multirow{6}{*}{12} & \multirow{3}{*}{ EUSAAR2 } & 8.09 & 26.94 & 18.10 & 98.62 & 8.62 & 16.26 & 28.54 \\
\hline & & 9.68 & 32.99 & 18.68 & 68.35 & 9.05 & 16.07 & 29.07 \\
\hline & & 10.05 & 29.40 & & & & & \\
\hline & \multirow{3}{*}{ NIOSH870 } & 6.75 & 32.79 & 25.78 & 73.93 & 8.33 & 16.54 & 31.04 \\
\hline & & 7.04 & 25.79 & 21.85 & 68.35 & 9.00 & 16.80 & 40.42 \\
\hline & & 7.16 & 32.25 & & & & & \\
\hline \multirow{6}{*}{13} & \multirow{3}{*}{ EUSAAR2 } & & 37.46 & 25.41 & 80.70 & 9.50 & 18.22 & 33.83 \\
\hline & & & 39.51 & 22.25 & 74.25 & 9.91 & 18.93 & 36.77 \\
\hline & & & 38.24 & & & & & \\
\hline & \multirow{3}{*}{ NIOSH870 } & & 39.81 & 23.19 & 79.40 & 10.05 & 19.97 & 36.45 \\
\hline & & & 39.16 & 22.76 & 78.39 & 9.56 & 21.09 & 34.28 \\
\hline & & & 38.80 & & & & & \\
\hline \multirow{6}{*}{14} & \multirow{3}{*}{ EUSAAR2 } & 9.71 & 32.76 & 19.63 & 78.88 & 9.41 & 16.70 & 31.90 \\
\hline & & 9.66 & 33.07 & 19.61 & 73.74 & 8.73 & 16.60 & 34.84 \\
\hline & & 9.46 & 32.94 & & & & & \\
\hline & \multirow{3}{*}{ NIOSH870 } & 10.36 & 33.76 & 19.86 & 73.42 & 8.71 & 16.64 & 33.70 \\
\hline & & 10.31 & 33.73 & 19.41 & 77.30 & 8.72 & 17.07 & 31.94 \\
\hline & & 10.36 & 34.61 & & & & & \\
\hline \multirow{6}{*}{15} & \multirow{3}{*}{ EUSAAR2 } & 10.01 & 32.24 & 20.09 & 79.30 & 9.09 & 16.88 & 31.94 \\
\hline & & 10.53 & 32.88 & 20.28 & 74.64 & 9.14 & 16.59 & 34.03 \\
\hline & & 10.36 & 33.91 & & & & & \\
\hline & \multirow{3}{*}{ NIOSH870 } & 10.93 & 33.46 & 21.02 & 76.45 & 9.47 & 17.15 & 34.11 \\
\hline & & 11.92 & 34.12 & 20.67 & 77.70 & 8.93 & 17.80 & \\
\hline & & 11.71 & 33.54 & & & & & \\
\hline \multirow{6}{*}{16} & \multirow{3}{*}{ EUSAAR2 } & 13.71 & 41.98 & 20.15 & 53.07 & 10.13 & 20.19 & 27.55 \\
\hline & & 13.93 & 37.58 & 18.37 & 87.61 & 11.20 & 18.75 & 27.01 \\
\hline & & 11.94 & 44.34 & & & & & \\
\hline & \multirow{3}{*}{ NIOSH870 } & 13.17 & 38.97 & 20.75 & 51.83 & 10.11 & 19.34 & 36.67 \\
\hline & & 12.61 & 42.24 & 19.57 & 47.83 & 16.28 & 20.24 & 33.13 \\
\hline & & 12.77 & 45.08 & & & & & \\
\hline \multirow{6}{*}{17} & \multirow{3}{*}{ EUSAAR2 } & 8.10 & 31.23 & 19.70 & & & 19.02 & \\
\hline & & 8.48 & 31.50 & 19.72 & & & 17.57 & \\
\hline & & 9.89 & 31.73 & & & & & \\
\hline & \multirow{3}{*}{ NIOSH870 } & 7.95 & 32.06 & 20.51 & & & 17.52 & \\
\hline & & 8.67 & 33.86 & 21.49 & & & 18.24 & \\
\hline & & 8.52 & 32.13 & & & & & \\
\hline
\end{tabular}


Table S3. EC (TOT) results per participant and protocol in $\mu \mathrm{g} / \mathrm{cm}^{2}$.

\begin{tabular}{|c|c|c|c|c|c|c|}
\hline Laboratory & EC & $\mathbf{A}$ & B & D & G & $\mathbf{U}$ \\
\hline \multirow{4}{*}{1} & \multirow{2}{*}{ EUSAAR2 } & 3.52 & 11.00 & 1.35 & 5.75 & 5.26 \\
\hline & & 3.47 & 11.10 & 1.28 & 5.06 & 4.49 \\
\hline & \multirow{2}{*}{ NIOSH870 } & 3.10 & 7.63 & 1.29 & 5.22 & 3.19 \\
\hline & & 3.13 & 7.24 & 1.00 & 4.82 & 3.00 \\
\hline \multirow{4}{*}{2} & \multirow{2}{*}{ EUSAAR2 } & 2.92 & 12.15 & 1.01 & 4.41 & 3.38 \\
\hline & & 2.92 & 12.57 & 0.94 & 4.28 & 3.56 \\
\hline & \multirow{2}{*}{ NIOSH870 } & 1.96 & 7.76 & 0.74 & 4.57 & 2.64 \\
\hline & & 2.08 & 8.39 & 0.67 & 5.18 & 2.33 \\
\hline \multirow{4}{*}{3} & \multirow{2}{*}{ EUSAAR2 } & 3.84 & 5.02 & 0.97 & 4.91 & 3.82 \\
\hline & & 3.62 & 4.48 & 1.35 & 4.88 & 3.60 \\
\hline & \multirow{2}{*}{ NIOSH930 } & 2.20 & 3.78 & 0.05 & 4.57 & 3.68 \\
\hline & & 2.48 & 4.41 & 0.01 & 4.65 & 3.58 \\
\hline \multirow{4}{*}{4} & \multirow{2}{*}{ EUSAAR2 } & 2.80 & 12.22 & 1.38 & 5.30 & 3.56 \\
\hline & & 2.71 & 13.81 & 1.16 & 5.16 & 3.73 \\
\hline & \multirow{2}{*}{ NIOSH870 } & 2.45 & 7.80 & 1.16 & 3.11 & 2.49 \\
\hline & & 2.47 & 7.41 & 1.15 & 3.06 & 2.28 \\
\hline \multirow{4}{*}{5} & \multirow{2}{*}{ EUSAAR2 } & 3.07 & 15.90 & 1.27 & 4.19 & 5.09 \\
\hline & & 3.32 & 14.44 & & 4.11 & 4.67 \\
\hline & \multirow{2}{*}{ NIOSH-like } & 2.24 & 12.22 & 0.95 & 4.26 & 3.77 \\
\hline & & 2.31 & 10.44 & 1.00 & 4.03 & 3.50 \\
\hline \multirow{4}{*}{6} & \multirow{2}{*}{ EUSAAR2 } & 3.19 & 11.27 & 1.32 & 4.45 & 4.20 \\
\hline & & 3.23 & 11.24 & 1.06 & 4.38 & 3.97 \\
\hline & \multirow{2}{*}{ NIOSH870 } & 1.71 & 5.73 & 0.72 & 4.10 & 1.96 \\
\hline & & 1.76 & 6.47 & 0.78 & 4.10 & 1.93 \\
\hline \multirow{8}{*}{7} & \multirow{4}{*}{ EUSAAR2 } & 4.23 & & & 4.41 & \\
\hline & & 3.61 & & & 4.27 & \\
\hline & & 3.64 & & & 4.20 & \\
\hline & & 3.63 & & & 4.37 & \\
\hline & \multirow{4}{*}{ NIOSH870 } & 2.36 & 10.86 & 0.94 & 4.48 & 2.84 \\
\hline & & 2.59 & 11.53 & 1.01 & 4.40 & 3.39 \\
\hline & & 2.27 & 10.28 & 0.94 & 4.23 & 3.27 \\
\hline & & 2.42 & 10.31 & 0.80 & 4.29 & 3.26 \\
\hline \multirow{4}{*}{8} & \multirow{2}{*}{ EUSAAR2 } & 4.42 & 13.72 & 1.64 & 4.94 & 4.82 \\
\hline & & 4.30 & 13.73 & 1.55 & 5.00 & 4.67 \\
\hline & \multirow{2}{*}{ NIOSH870 } & 3.73 & 10.14 & 1.21 & 5.43 & 3.61 \\
\hline & & 3.78 & 11.48 & 1.42 & 5.48 & 3.11 \\
\hline
\end{tabular}


Table S3 (continued). EC (TOT) results per participant and protocol in $\mu \mathrm{g} / \mathrm{cm}^{2}$.

\begin{tabular}{|c|c|c|c|c|c|c|}
\hline Laboratory & EC & A & B & D & G & $\mathbf{U}$ \\
\hline \multirow{4}{*}{9} & \multirow{2}{*}{ EUSAAR2 } & 3.55 & 12.01 & 1.31 & 4.45 & 5.40 \\
\hline & & 3.66 & 11.50 & 1.49 & 5.05 & 4.24 \\
\hline & \multirow{2}{*}{ NIOSH870 } & 2.91 & 6.28 & 0.85 & 4.40 & 2.91 \\
\hline & & 2.95 & 6.92 & 0.90 & 4.62 & 2.93 \\
\hline \multirow{4}{*}{10} & \multirow{2}{*}{ EUSAAR2 } & 1.18 & 8.36 & -0.36 & 1.97 & 2.29 \\
\hline & & 1.85 & 6.89 & 0.20 & 1.65 & 1.68 \\
\hline & \multirow{2}{*}{ NIOSH-like } & 0.85 & 5.74 & 0.55 & 1.16 & 2.04 \\
\hline & & 2.66 & 8.33 & 0.12 & 1.65 & 1.34 \\
\hline \multirow{4}{*}{11} & \multirow{2}{*}{ EUSAAR2 } & 4.13 & 14.92 & 1.75 & 6.86 & 6.45 \\
\hline & & 4.03 & 16.60 & 1.56 & 5.65 & 6.45 \\
\hline & \multirow{2}{*}{ NIOSH870 } & 3.71 & 17.49 & 1.36 & 6.06 & 4.86 \\
\hline & & 3.54 & 17.86 & 1.46 & & 4.83 \\
\hline \multirow{4}{*}{12} & \multirow{2}{*}{ EUSAAR2 } & 2.69 & 8.95 & 1.37 & 4.06 & 3.54 \\
\hline & & 3.31 & 7.63 & 1.50 & 3.63 & 4.08 \\
\hline & \multirow{2}{*}{ NIOSH870 } & 3.98 & 7.27 & 0.90 & 3.59 & 2.84 \\
\hline & & 3.06 & 8.17 & 0.87 & 3.80 & 4.41 \\
\hline \multirow{4}{*}{13} & \multirow{2}{*}{ EUSAAR2 } & 2.08 & 8.87 & 0.97 & 4.47 & 2.25 \\
\hline & & 2.35 & 7.84 & 1.07 & 4.57 & 2.52 \\
\hline & \multirow{2}{*}{ NIOSH870 } & 2.24 & 6.34 & 0.82 & 5.21 & 2.44 \\
\hline & & 2.45 & 6.67 & 0.66 & 5.41 & 2.11 \\
\hline \multirow{4}{*}{14} & \multirow{2}{*}{ EUSAAR2 } & 3.51 & 11.24 & 1.36 & 4.61 & 3.93 \\
\hline & & 3.44 & 10.26 & 1.33 & 4.55 & 4.66 \\
\hline & \multirow{2}{*}{ NIOSH870 } & 2.99 & 8.77 & 1.03 & 4.38 & 2.75 \\
\hline & & 2.84 & 9.59 & 1.04 & 3.89 & 2.30 \\
\hline \multirow{4}{*}{15} & \multirow{2}{*}{ EUSAAR2 } & 3.56 & 14.14 & 1.52 & 4.75 & 4.63 \\
\hline & & 3.49 & 12.95 & 0.74 & 4.56 & 1.14 \\
\hline & \multirow{2}{*}{ NIOSH870 } & 2.28 & 7.79 & 0.89 & 4.57 & 2.77 \\
\hline & & 2.48 & 7.35 & 0.93 & 5.40 & \\
\hline \multirow{4}{*}{16} & \multirow{2}{*}{ EUSAAR2 } & 3.00 & 11.69 & 1.35 & 4.42 & 4.42 \\
\hline & & 3.10 & 19.11 & 1.41 & 4.48 & 3.67 \\
\hline & \multirow{2}{*}{ NIOSH870 } & 1.86 & 4.73 & 0.94 & 5.07 & 0.68 \\
\hline & & 1.99 & 3.76 & 1.13 & 5.59 & 2.43 \\
\hline \multirow{4}{*}{17} & \multirow{2}{*}{ EUSAAR2 } & 3.24 & & & 4.57 & \\
\hline & & 2.97 & & & 4.36 & \\
\hline & \multirow{2}{*}{ NIOSH870 } & 2.68 & & & 5.78 & \\
\hline & & 3.40 & & & 4.80 & \\
\hline
\end{tabular}


Table S4. EC/TC (TOT) ratios results per participant and protocol.

\begin{tabular}{|c|c|c|c|c|c|c|}
\hline Laboratory & EC/TC & A & B & D & $\mathbf{G}$ & $\mathbf{U}$ \\
\hline \multirow{4}{*}{1} & \multirow{2}{*}{ EUSAAR2 } & 0.17 & 0.15 & 0.16 & 0.33 & 0.16 \\
\hline & & 0.17 & 0.15 & 0.16 & 0.30 & 0.14 \\
\hline & \multirow{2}{*}{ NIOSH870 } & 0.15 & 0.10 & 0.13 & 0.30 & 0.10 \\
\hline & & 0.15 & 0.10 & 0.11 & 0.28 & 0.09 \\
\hline \multirow{4}{*}{2} & \multirow{2}{*}{ EUSAAR2 } & 0.14 & 0.16 & 0.11 & 0.25 & 0.10 \\
\hline & & 0.14 & 0.16 & 0.10 & 0.25 & 0.11 \\
\hline & \multirow{2}{*}{ NIOSH870 } & 0.09 & 0.10 & 0.07 & 0.25 & 0.07 \\
\hline & & 0.10 & 0.12 & 0.07 & 0.28 & 0.07 \\
\hline \multirow{4}{*}{3} & \multirow{2}{*}{ EUSAAR2 } & 0.15 & 0.06 & 0.10 & 0.28 & 0.11 \\
\hline & & 0.17 & 0.06 & 0.15 & 0.27 & 0.12 \\
\hline & \multirow{2}{*}{ NIOSH930 } & 0.10 & 0.05 & 0.01 & 0.24 & 0.12 \\
\hline & & 0.11 & 0.06 & 0.00 & 0.25 & 0.12 \\
\hline \multirow{4}{*}{4} & \multirow{2}{*}{ EUSAAR2 } & 0.15 & 0.18 & 0.15 & 0.30 & 0.12 \\
\hline & & 0.15 & 0.19 & 0.12 & 0.29 & 0.11 \\
\hline & \multirow{2}{*}{ NIOSH870 } & 0.13 & 0.11 & 0.12 & 0.18 & 0.08 \\
\hline & & 0.13 & 0.11 & 0.12 & 0.18 & 0.08 \\
\hline \multirow{4}{*}{5} & \multirow{2}{*}{ EUSAAR2 } & 0.16 & 0.21 & 0.14 & 0.25 & 0.16 \\
\hline & & 0.16 & 0.20 & & 0.25 & 0.15 \\
\hline & \multirow{2}{*}{ NIOSH-like } & 0.11 & 0.16 & 0.10 & 0.24 & 0.11 \\
\hline & & 0.11 & 0.14 & 0.10 & 0.23 & 0.10 \\
\hline \multirow{4}{*}{6} & \multirow{2}{*}{ EUSAAR2 } & 0.15 & 0.13 & 0.13 & 0.26 & 0.11 \\
\hline & & 0.15 & 0.14 & 0.11 & 0.25 & 0.11 \\
\hline & \multirow{2}{*}{ NIOSH870 } & 0.08 & 0.08 & 0.08 & 0.23 & 0.06 \\
\hline & & 0.08 & 0.08 & 0.08 & 0.23 & 0.06 \\
\hline \multirow{8}{*}{7} & \multirow{4}{*}{ EUSAAR2 } & 0.20 & & & 0.25 & \\
\hline & & 0.19 & & & 0.25 & \\
\hline & & 0.20 & & & 0.25 & \\
\hline & & 0.19 & & & 0.25 & \\
\hline & \multirow{4}{*}{ NIOSH870 } & 0.12 & 0.15 & 0.10 & 0.27 & 0.09 \\
\hline & & 0.13 & 0.15 & 0.11 & 0.25 & 0.10 \\
\hline & & 0.12 & 0.15 & 0.10 & 0.25 & 0.10 \\
\hline & & 0.12 & 0.14 & 0.09 & 0.25 & 0.10 \\
\hline \multirow{4}{*}{8} & \multirow{2}{*}{ EUSAAR2 } & 0.22 & 0.18 & 0.17 & 0.28 & 0.13 \\
\hline & & 0.21 & 0.18 & 0.17 & 0.29 & 0.14 \\
\hline & \multirow{2}{*}{ NIOSH870 } & 0.18 & 0.14 & 0.13 & 0.30 & 0.10 \\
\hline & & 0.19 & 0.15 & 0.15 & 0.29 & 0.09 \\
\hline
\end{tabular}


Table S4 (Continued). EC/TC (TOT) ratios results per participant and protocol.

\begin{tabular}{|c|c|c|c|c|c|c|}
\hline Laboratory & EC/TC & $\mathbf{A}$ & B & D & $\mathbf{G}$ & $\mathbf{U}$ \\
\hline \multirow{4}{*}{9} & \multirow{2}{*}{ EUSAAR2 } & 0.17 & 0.16 & 0.11 & 0.27 & 0.14 \\
\hline & & 0.17 & 0.15 & 0.16 & 0.27 & 0.12 \\
\hline & \multirow{2}{*}{ NIOSH870 } & 0.14 & 0.09 & 0.09 & 0.26 & 0.09 \\
\hline & & 0.15 & 0.10 & 0.09 & 0.26 & 0.10 \\
\hline \multirow{4}{*}{10} & \multirow{2}{*}{ EUSAAR2 } & 0.04 & 0.09 & -0.04 & 0.08 & 0.05 \\
\hline & & 0.07 & 0.07 & 0.02 & 0.07 & 0.04 \\
\hline & \multirow{2}{*}{ NIOSH-like } & 0.03 & 0.06 & 0.04 & 0.05 & 0.04 \\
\hline & & 0.10 & 0.09 & 0.01 & 0.07 & 0.03 \\
\hline \multirow{4}{*}{11} & \multirow{2}{*}{ EUSAAR2 } & 0.14 & 0.14 & 0.14 & 0.28 & 0.12 \\
\hline & & 0.14 & 0.16 & 0.13 & 0.24 & 0.12 \\
\hline & \multirow{2}{*}{ NIOSH870 } & 0.12 & 0.15 & 0.10 & 0.22 & 0.10 \\
\hline & & 0.11 & 0.16 & 0.11 & & 0.10 \\
\hline \multirow{4}{*}{12} & \multirow{2}{*}{ EUSAAR2 } & 0.15 & 0.09 & 0.16 & 0.25 & 0.12 \\
\hline & & 0.18 & 0.11 & 0.17 & 0.23 & 0.14 \\
\hline & \multirow{2}{*}{ NIOSH870 } & 0.15 & 0.10 & 0.11 & 0.22 & 0.09 \\
\hline & & 0.14 & 0.12 & 0.10 & 0.23 & 0.11 \\
\hline \multirow{4}{*}{13} & \multirow{2}{*}{ EUSAAR2 } & 0.08 & 0.11 & 0.10 & 0.25 & 0.07 \\
\hline & & 0.11 & 0.11 & 0.11 & 0.24 & 0.07 \\
\hline & \multirow{2}{*}{ NIOSH870 } & 0.10 & 0.08 & 0.08 & 0.26 & 0.07 \\
\hline & & 0.11 & 0.09 & 0.07 & 0.26 & 0.06 \\
\hline \multirow{4}{*}{14} & \multirow{2}{*}{ EUSAAR2 } & 0.18 & 0.14 & 0.14 & 0.28 & 0.12 \\
\hline & & 0.18 & 0.14 & 0.15 & 0.27 & 0.13 \\
\hline & \multirow{2}{*}{ NIOSH870 } & 0.15 & 0.12 & 0.12 & 0.26 & 0.08 \\
\hline & & 0.15 & 0.12 & 0.12 & 0.23 & 0.07 \\
\hline \multirow{4}{*}{15} & \multirow{2}{*}{ EUSAAR2 } & 0.18 & 0.18 & 0.17 & 0.28 & 0.14 \\
\hline & & 0.17 & 0.17 & 0.08 & 0.27 & 0.03 \\
\hline & \multirow{2}{*}{ NIOSH870 } & 0.11 & 0.10 & 0.09 & 0.27 & 0.08 \\
\hline & & 0.12 & 0.09 & 0.10 & 0.30 & \\
\hline \multirow{4}{*}{16} & \multirow{2}{*}{ EUSAAR2 } & 0.15 & 0.22 & 0.13 & 0.22 & 0.16 \\
\hline & & 0.17 & 0.22 & 0.13 & 0.24 & 0.14 \\
\hline & \multirow{2}{*}{ NIOSH870 } & 0.09 & 0.09 & 0.09 & 0.26 & 0.02 \\
\hline & & 0.10 & 0.08 & 0.07 & 0.28 & 0.07 \\
\hline \multirow{4}{*}{17} & \multirow{2}{*}{ EUSAAR2 } & 0.16 & & & 0.24 & \\
\hline & & 0.15 & & & 0.25 & \\
\hline & \multirow{2}{*}{ NIOSH870 } & 0.13 & & & 0.33 & \\
\hline & & 0.16 & & & 0.26 & \\
\hline
\end{tabular}


Table S5. OC (TOT) results per participant and protocol in $\mu \mathrm{g} / \mathrm{cm}^{2}$.

\begin{tabular}{|c|c|c|c|c|c|c|}
\hline Laboratory & $\mathrm{OC}$ & $\mathbf{A}$ & B & D & G & $\mathbf{U}$ \\
\hline \multirow{4}{*}{1} & \multirow{2}{*}{ EUSAAR2 } & 17.23 & 62.56 & 7.14 & 11.57 & 28.66 \\
\hline & & 16.97 & 62.55 & 6.82 & 11.60 & 27.48 \\
\hline & \multirow{2}{*}{ NIOSH870 } & 17.41 & 65.92 & 8.36 & 12.23 & 28.25 \\
\hline & & 17.77 & 65.25 & 8.11 & 12.55 & 29.28 \\
\hline \multirow{4}{*}{2} & \multirow{2}{*}{ EUSAAR2 } & 17.66 & 64.46 & 8.15 & 13.19 & 32.19 \\
\hline & & 17.75 & 67.64 & 8.13 & 12.88 & 29.82 \\
\hline & \multirow{2}{*}{ NIOSH870 } & 19.50 & 67.28 & 9.83 & 13.87 & 33.29 \\
\hline & & 19.15 & 64.27 & 8.77 & 13.14 & 30.15 \\
\hline \multirow{4}{*}{3} & \multirow{2}{*}{ EUSAAR2 } & 22.20 & 73.59 & 9.23 & 12.93 & 29.49 \\
\hline & & 17.49 & 68.92 & 7.83 & 13.07 & 26.81 \\
\hline & \multirow{2}{*}{ NIOSH930 } & 19.34 & 69.91 & 9.86 & 14.18 & 27.18 \\
\hline & & 20.03 & 72.40 & 9.44 & 13.85 & 25.84 \\
\hline \multirow{4}{*}{4} & \multirow{2}{*}{ EUSAAR2 } & 15.92 & 56.70 & 7.88 & 12.36 & 27.36 \\
\hline & & 15.68 & 60.24 & 8.71 & 12.39 & 28.86 \\
\hline & \multirow{2}{*}{ NIOSH870 } & 16.26 & 60.90 & 8.76 & 14.22 & 29.40 \\
\hline & & 16.00 & 59.85 & 8.08 & 14.23 & 27.52 \\
\hline \multirow{4}{*}{5} & \multirow{2}{*}{ EUSAAR2 } & 16.18 & 61.58 & 7.84 & 12.30 & 27.04 \\
\hline & & 17.44 & 58.60 & & 12.65 & 27.34 \\
\hline & \multirow{2}{*}{ NIOSH-like } & 17.93 & 62.76 & 8.74 & 13.28 & 30.71 \\
\hline & & 18.19 & 62.08 & 8.83 & 13.43 & 30.12 \\
\hline \multirow{4}{*}{6} & \multirow{2}{*}{ EUSAAR2 } & 18.16 & 75.67 & 8.73 & 12.98 & 33.41 \\
\hline & & 18.44 & 68.63 & 8.48 & 13.17 & 32.18 \\
\hline & \multirow{2}{*}{ NIOSH870 } & 19.64 & 67.14 & 8.55 & 14.00 & 32.84 \\
\hline & & 19.40 & 70.95 & 8.51 & 13.65 & 31.29 \\
\hline \multirow{8}{*}{7} & \multirow{4}{*}{ EUSAAR2 } & 17.02 & & & 13.39 & \\
\hline & & 15.35 & & & 12.67 & \\
\hline & & 15.01 & & & 12.43 & \\
\hline & & 15.21 & & & 13.11 & \\
\hline & \multirow{4}{*}{ NIOSH870 } & 17.82 & 62.29 & 8.08 & 12.39 & 28.01 \\
\hline & & 17.99 & 63.24 & 8.26 & 12.90 & 29.46 \\
\hline & & 16.64 & 60.02 & 8.56 & 12.97 & 29.47 \\
\hline & & 17.96 & 61.55 & 8.37 & 13.04 & 29.86 \\
\hline \multirow{4}{*}{8} & \multirow{2}{*}{ EUSAAR2 } & 15.63 & 62.58 & 7.87 & 12.95 & 31.30 \\
\hline & & 15.77 & 64.34 & 7.69 & $\overline{12.42}$ & 29.64 \\
\hline & \multirow{2}{*}{ NIOSH870 } & 16.49 & 64.48 & 8.35 & 12.45 & 30.87 \\
\hline & & 16.49 & 67.38 & 8.08 & 13.15 & 30.75 \\
\hline
\end{tabular}


Table S5 (continued). OC (TOT) results per participant and protocol in $\mu \mathrm{g} / \mathrm{cm}^{2}$.

\begin{tabular}{|c|c|c|c|c|c|c|}
\hline Laboratory & OC & $\mathbf{A}$ & B & D & G & $\mathbf{U}$ \\
\hline \multirow{4}{*}{9} & \multirow{2}{*}{ EUSAAR2 } & 17.54 & 63.56 & 10.48 & 12.07 & 33.54 \\
\hline & & 17.40 & 65.05 & 8.00 & 13.55 & 30.66 \\
\hline & \multirow{2}{*}{ NIOSH870 } & 17.61 & 62.31 & 8.19 & 12.35 & 27.76 \\
\hline & & 17.19 & 62.55 & 9.07 & 12.97 & 27.90 \\
\hline \multirow{4}{*}{10} & \multirow{2}{*}{ EUSAAR2 } & 25.23 & 86.43 & 10.20 & 21.80 & 40.21 \\
\hline & & 24.28 & 86.34 & 10.94 & 22.93 & 38.80 \\
\hline & \multirow{2}{*}{ NIOSH-like } & 29.56 & 87.62 & 14.93 & 24.05 & 45.16 \\
\hline & & 24.60 & 87.34 & 12.14 & 23.48 & 45.43 \\
\hline \multirow{4}{*}{11} & \multirow{2}{*}{ EUSAAR2 } & 25.62 & 90.98 & 10.43 & 18.03 & 45.51 \\
\hline & & 25.65 & 88.32 & 10.25 & 18.30 & 45.53 \\
\hline & \multirow{2}{*}{ NIOSH870 } & 27.04 & 97.09 & 12.45 & 21.93 & 41.72 \\
\hline & & 27.28 & 92.18 & 12.23 & & 43.00 \\
\hline \multirow{4}{*}{12} & \multirow{2}{*}{ EUSAAR2 } & 15.41 & 89.67 & 7.25 & 12.20 & 25.00 \\
\hline & & 15.37 & 60.72 & 7.55 & 12.44 & 24.99 \\
\hline & \multirow{2}{*}{ NIOSH870 } & 21.80 & 66.66 & 7.43 & 12.95 & 28.20 \\
\hline & & 18.79 & 60.18 & 8.13 & 13.00 & 36.01 \\
\hline \multirow{4}{*}{13} & \multirow{2}{*}{ EUSAAR2 } & 23.33 & 71.83 & 8.53 & 13.75 & 31.58 \\
\hline & & 19.90 & 66.41 & 8.84 & 14.36 & 34.25 \\
\hline & \multirow{2}{*}{ NIOSH870 } & 20.95 & 73.06 & 9.23 & 14.76 & 34.01 \\
\hline & & 20.31 & 71.72 & 8.90 & 15.68 & 32.17 \\
\hline \multirow{4}{*}{14} & \multirow{2}{*}{ EUSAAR2 } & 16.12 & 67.64 & 8.05 & 12.09 & 27.97 \\
\hline & & 16.17 & 63.48 & 7.40 & 12.05 & 30.18 \\
\hline & \multirow{2}{*}{ NIOSH870 } & 16.87 & 64.65 & 7.68 & 12.26 & 30.95 \\
\hline & & 16.57 & 67.71 & 7.68 & 13.18 & 29.64 \\
\hline \multirow{4}{*}{15} & \multirow{2}{*}{ EUSAAR2 } & 16.53 & 65.16 & 7.57 & 12.13 & 27.31 \\
\hline & & 16.79 & 61.69 & 8.40 & 12.03 & 32.89 \\
\hline & \multirow{2}{*}{ NIOSH870 } & 18.74 & 68.66 & 8.58 & 12.58 & 31.34 \\
\hline & & 18.19 & 70.35 & 8.00 & 12.40 & \\
\hline \multirow{4}{*}{16} & \multirow{2}{*}{ EUSAAR2 } & 17.15 & 41.38 & 8.78 & 15.77 & 23.13 \\
\hline & & 15.27 & 68.50 & 9.79 & 14.27 & 23.34 \\
\hline & \multirow{2}{*}{ NIOSH870 } & 18.89 & 47.10 & 9.17 & 14.27 & 35.99 \\
\hline & & 17.58 & 44.07 & 15.15 & 14.65 & 30.70 \\
\hline \multirow{4}{*}{17} & \multirow{2}{*}{ EUSAAR2 } & 16.46 & & & 14.45 & \\
\hline & & 16.75 & & & 13.21 & \\
\hline & \multirow{2}{*}{ NIOSH870 } & 17.83 & & & 11.74 & \\
\hline & & 18.09 & & & 13.44 & \\
\hline
\end{tabular}


Table S6. EC (TOR) results per participant and protocol in $\mu \mathrm{g} / \mathrm{cm}^{2}$.

\begin{tabular}{|c|c|c|c|c|c|c|}
\hline Laboratory & EC & $\mathbf{A}$ & B & D & $\mathbf{G}$ & $\mathbf{U}$ \\
\hline \multirow{4}{*}{1} & \multirow{2}{*}{ EUSAAR2 } & 2.90 & 10.64 & 0.64 & 3.52 & 6.31 \\
\hline & & 3.19 & 8.79 & 0.69 & 2.88 & 6.10 \\
\hline & \multirow{2}{*}{ NIOSH870 } & 1.63 & 9.07 & 0.80 & 2.58 & 5.29 \\
\hline & & 1.92 & 7.75 & 0.78 & 1.63 & 4.61 \\
\hline \multirow{4}{*}{2} & \multirow{2}{*}{ EUSAAR2 } & 3.62 & 24.49 & 2.06 & 5.04 & 10.67 \\
\hline & & 3.34 & 25.11 & 1.97 & 5.30 & 10.08 \\
\hline & \multirow{2}{*}{ NIOSH870 } & 3.29 & 21.58 & 1.49 & 4.92 & 8.92 \\
\hline & & 2.81 & 21.61 & 1.58 & 4.82 & 9.32 \\
\hline \multirow{4}{*}{3} & \multirow{2}{*}{ EUSAAR2 } & 4.99 & 11.06 & 1.74 & 3.88 & 0.17 \\
\hline & & 5.27 & 11.81 & 0.00 & 2.84 & 1.89 \\
\hline & \multirow{2}{*}{ NIOSH930 } & 0.00 & 0.01 & 1.79 & 0.00 & 0.02 \\
\hline & & 0.00 & 0.00 & 1.41 & 0.00 & 2.23 \\
\hline \multirow{4}{*}{5} & \multirow{2}{*}{ EUSAAR2 } & 3.12 & 20.69 & 1.80 & 4.08 & 8.32 \\
\hline & & 3.12 & 18.76 & & 3.81 & 8.50 \\
\hline & \multirow{2}{*}{ NIOSH-like } & 2.71 & 16.97 & 1.38 & 3.99 & 7.60 \\
\hline & & 2.90 & 15.04 & 1.80 & 3.91 & 7.25 \\
\hline \multirow{4}{*}{6} & \multirow{2}{*}{ EUSAAR2 } & 4.03 & 29.14 & 2.99 & 5.76 & 11.63 \\
\hline & & 4.15 & 28.01 & 2.56 & 5.62 & 11.76 \\
\hline & \multirow{2}{*}{ NIOSH870 } & 2.27 & 18.94 & 1.71 & 5.01 & 8.17 \\
\hline & & 2.45 & 19.61 & 1.62 & 4.83 & 8.00 \\
\hline \multirow{8}{*}{7} & \multirow{4}{*}{ EUSAAR2 } & 4.71 & & & 5.62 & \\
\hline & & 4.23 & & & 5.45 & \\
\hline & & 4.29 & & & 5.62 & \\
\hline & & 4.25 & & & 5.63 & \\
\hline & \multirow{4}{*}{ NIOSH870 } & 3.50 & 24.49 & 1.72 & 4.79 & 8.22 \\
\hline & & 3.78 & 23.67 & 1.82 & 4.95 & 9.24 \\
\hline & & 3.37 & 23.86 & 1.57 & 4.80 & 10.05 \\
\hline & & 3.69 & 22.37 & 1.66 & 4.99 & 10.00 \\
\hline \multirow{4}{*}{8} & \multirow{2}{*}{ EUSAAR2 } & 6.92 & 14.43 & 1.99 & 2.88 & 13.47 \\
\hline & & 3.82 & 7.59 & 2.22 & 4.53 & 8.83 \\
\hline & \multirow{2}{*}{ NIOSH870 } & 3.08 & 22.47 & 1.48 & 3.81 & 19.22 \\
\hline & & 3.37 & 18.07 & 2.16 & 4.72 & 10.71 \\
\hline
\end{tabular}


Table S6 (continued). EC (TOR) results per participant and protocol in $\mu \mathrm{g} / \mathrm{cm}^{2}$.

\begin{tabular}{|c|c|c|c|c|c|c|}
\hline Laboratory & $\mathbf{O C}$ & $\mathbf{A}$ & $\mathbf{B}$ & $\mathbf{D}$ & $\mathbf{G}$ & $\mathbf{U}$ \\
\hline \multirow{3}{*}{$\mathbf{1 0}$} & \multirow{2}{*}{ EUSAAR2 } & 1.65 & 9.65 & 0.23 & 0.94 & 4.74 \\
\cline { 3 - 7 } & & 1.53 & 9.09 & 0.46 & 1.05 & 4.41 \\
\cline { 3 - 7 } & \multirow{2}{*}{ NIOSH-like } & 1.42 & 10.47 & 1.23 & 1.11 & 5.49 \\
\cline { 3 - 7 } & & 1.55 & 14.69 & 0.05 & 1.58 & 5.83 \\
\hline \multirow{3}{*}{$\mathbf{1 3}$} & \multirow{2}{*}{ EUSAAR2 } & 2.08 & 7.81 & 1.26 & 2.57 & 5.37 \\
\cline { 3 - 7 } & & 2.21 & 7.19 & 1.37 & 2.71 & 6.01 \\
\cline { 2 - 7 } & \multirow{2}{*}{ NIOSH870 } & 2.09 & 8.03 & 0.73 & 2.26 & 7.33 \\
\cline { 3 - 7 } & & 2.02 & 10.20 & 0.97 & 2.61 & 5.78 \\
\hline \multirow{3}{*}{$\mathbf{1 4}$} & \multirow{2}{*}{ EUSAAR2 } & 4.31 & 16.15 & 2.61 & 2.69 & 6.51 \\
\cline { 3 - 7 } & & 3.09 & 12.62 & 1.48 & 3.39 & 9.46 \\
\cline { 2 - 7 } & \multirow{2}{*}{ NIOSH870 } & 3.94 & 9.42 & 1.03 & 4.07 & 8.57 \\
\cline { 3 - 7 } & & 2.19 & 26.91 & 1.29 & 0.00 & 5.04 \\
\hline
\end{tabular}


Table S7. EC/TC (TOR) ratios per participant and protocol.

\begin{tabular}{|c|c|c|c|c|c|c|}
\hline Laboratory & EC/TC & $\mathbf{A}$ & B & D & $\mathbf{G}$ & $\mathbf{U}$ \\
\hline \multirow{4}{*}{1} & \multirow{2}{*}{ EUSAAR2 } & 0.14 & 0.14 & 0.07 & 0.20 & 0.19 \\
\hline & & 0.16 & 0.12 & 0.08 & 0.17 & 0.19 \\
\hline & \multirow{2}{*}{ NIOSH870 } & 0.08 & 0.12 & 0.08 & 0.15 & 0.17 \\
\hline & & 0.09 & 0.11 & 0.09 & 0.09 & 0.14 \\
\hline \multirow{4}{*}{2} & \multirow{2}{*}{ EUSAAR2 } & 0.18 & 0.32 & 0.22 & 0.29 & 0.30 \\
\hline & & 0.16 & 0.31 & 0.22 & 0.31 & 0.30 \\
\hline & \multirow{2}{*}{ NIOSH870 } & 0.15 & 0.29 & 0.14 & 0.27 & 0.25 \\
\hline & & 0.13 & 0.30 & 0.17 & 0.26 & 0.29 \\
\hline \multirow{4}{*}{3} & \multirow{2}{*}{ EUSAAR2 } & 0.19 & 0.14 & 0.17 & 0.22 & 0.01 \\
\hline & & 0.25 & 0.16 & $(0.00)$ & 0.16 & 0.06 \\
\hline & \multirow{2}{*}{ NIOSH930 } & $(0.00)$ & 0.00 & 0.18 & 0.00 & 0.00 \\
\hline & & 0.00 & - & 0.15 & 0.00 & 0.08 \\
\hline \multirow{4}{*}{5} & \multirow{2}{*}{ EUSAAR2 } & 0.16 & 0.27 & 0.20 & 0.25 & 0.26 \\
\hline & & 0.15 & 0.26 & & 0.23 & 0.27 \\
\hline & \multirow{2}{*}{ NIOSH-like } & 0.13 & 0.23 & 0.14 & 0.23 & 0.22 \\
\hline & & 0.14 & 0.21 & 0.18 & 0.22 & 0.22 \\
\hline \multirow{4}{*}{6} & \multirow{2}{*}{ EUSAAR2 } & 0.19 & 0.34 & 0.30 & 0.33 & 0.31 \\
\hline & & 0.19 & 0.35 & 0.27 & 0.32 & 0.33 \\
\hline & \multirow{2}{*}{ NIOSH870 } & 0.11 & 0.26 & 0.18 & 0.28 & 0.23 \\
\hline & & 0.12 & 0.25 & 0.17 & 0.27 & 0.24 \\
\hline \multirow{8}{*}{7} & \multirow{4}{*}{ EUSAAR2 } & 0.22 & & & 0.32 & \\
\hline & & 0.22 & & & 0.32 & \\
\hline & & 0.23 & & & 0.34 & \\
\hline & & 0.23 & & & 0.32 & \\
\hline & \multirow{4}{*}{ NIOSH870 } & 0.17 & 0.33 & 0.19 & 0.28 & 0.27 \\
\hline & & 0.18 & 0.32 & 0.20 & 0.29 & 0.28 \\
\hline & & 0.18 & 0.34 & 0.16 & 0.28 & 0.31 \\
\hline & & 0.18 & 0.31 & 0.18 & 0.29 & 0.30 \\
\hline \multirow{4}{*}{8} & \multirow{2}{*}{ EUSAAR2 } & 0.35 & 0.19 & 0.21 & 0.16 & 0.37 \\
\hline & & 0.19 & 0.10 & 0.24 & 0.26 & 0.26 \\
\hline & \multirow{2}{*}{ NIOSH870 } & 0.15 & 0.30 & 0.15 & 0.21 & 0.56 \\
\hline & & 0.17 & 0.23 & 0.23 & 0.25 & 0.32 \\
\hline
\end{tabular}


Table S7 (continued). EC/TC (TOR) ratios per participant and protocol.

\begin{tabular}{|c|c|c|c|c|c|c|}
\hline Laboratory & $\mathbf{O C}$ & $\mathbf{A}$ & $\mathbf{B}$ & $\mathbf{D}$ & $\mathbf{G}$ & $\mathbf{U}$ \\
\hline \multirow{3}{*}{10} & \multirow{2}{*}{ EUSAAR2 } & 0.06 & 0.10 & 0.02 & 0.04 & 0.11 \\
\cline { 3 - 7 } & & 0.06 & 0.10 & 0.04 & 0.04 & 0.11 \\
\cline { 2 - 7 } & \multirow{2}{*}{ NIOSH-like } & 0.05 & 0.11 & 0.08 & 0.04 & 0.12 \\
\cline { 3 - 7 } & & 0.06 & 0.15 & 0.00 & 0.06 & 0.12 \\
\hline \multirow{3}{*}{$\mathbf{1 3}$} & \multirow{2}{*}{ EUSAAR2 } & 0.08 & 0.10 & 0.13 & 0.14 & 0.16 \\
\cline { 3 - 7 } & \multirow{2}{*}{ NIOSH870 } & 0.10 & 0.10 & 0.14 & 0.14 & 0.16 \\
\cline { 3 - 7 } & & 0.09 & 0.10 & 0.07 & 0.11 & 0.20 \\
\hline \multirow{3}{*}{$\mathbf{1 4}$} & \multirow{2}{*}{ EUSAAR2 } & 0.22 & 0.13 & 0.10 & 0.12 & 0.17 \\
\cline { 3 - 7 } & & 0.16 & 0.17 & 0.17 & 0.20 & 0.27 \\
\cline { 3 - 7 } & \multirow{2}{*}{ NIOSH870 } & 0.20 & 0.13 & 0.12 & 0.24 & 0.25 \\
\cline { 3 - 7 } & & 0.11 & 0.35 & 0.15 & $(0.00)$ & 0.16 \\
\hline
\end{tabular}


Table S8. OC (TOR) results per participant and protocol in $\mu \mathrm{g} / \mathrm{cm}^{2}$.

\begin{tabular}{|c|c|c|c|c|c|c|}
\hline Laboratory & $\mathrm{OC}$ & $\mathbf{A}$ & B & D & $\mathbf{G}$ & $\mathbf{U}$ \\
\hline \multirow{4}{*}{1} & \multirow{2}{*}{ EUSAAR2 } & 17.85 & 62.92 & 7.85 & 13.80 & 27.61 \\
\hline & & 17.25 & 64.86 & 7.41 & 13.78 & 25.87 \\
\hline & \multirow{2}{*}{ NIOSH870 } & 18.88 & 64.48 & 8.85 & 14.87 & 26.15 \\
\hline & & 18.98 & 64.74 & 8.33 & 15.74 & 27.67 \\
\hline \multirow{4}{*}{2} & \multirow{2}{*}{ EUSAAR2 } & 16.96 & 52.12 & 7.10 & 12.56 & 24.90 \\
\hline & & 17.33 & 55.10 & 7.10 & 11.86 & 23.30 \\
\hline & \multirow{2}{*}{ NIOSH870 } & 18.17 & 53.46 & 9.08 & 13.52 & 27.01 \\
\hline & & 18.42 & 51.05 & 7.86 & 13.50 & 23.16 \\
\hline \multirow{4}{*}{3} & \multirow{2}{*}{ EUSAAR2 } & 21.05 & 67.55 & 8.46 & 13.96 & 33.14 \\
\hline & & 15.84 & 61.59 & 9.18 & 15.11 & 28.52 \\
\hline & \multirow{2}{*}{ NIOSH930 } & 21.54 & 73.68 & 8.12 & 18.75 & 30.84 \\
\hline & & 22.51 & 76.81 & 8.04 & 18.50 & 27.19 \\
\hline \multirow{4}{*}{4} & \multirow{2}{*}{ EUSAAR2 } & 16.13 & 56.79 & 7.31 & 12.41 & 23.81 \\
\hline & & 17.64 & 54.28 & & 12.95 & 23.51 \\
\hline & \multirow{2}{*}{ NIOSH-like } & 17.46 & 58.01 & 8.31 & 13.55 & 26.88 \\
\hline & & 17.60 & 57.48 & 8.03 & 13.55 & 26.37 \\
\hline \multirow{4}{*}{6} & \multirow{2}{*}{ EUSAAR2 } & 17.32 & 57.80 & 7.06 & 11.67 & 25.98 \\
\hline & & 17.52 & 51.86 & 6.98 & 11.93 & 24.39 \\
\hline & \multirow{2}{*}{ NIOSH870 } & 19.08 & 53.93 & 7.56 & 13.09 & 26.63 \\
\hline & & 18.71 & 57.81 & 7.67 & 12.92 & 25.22 \\
\hline \multirow{8}{*}{7} & \multirow{4}{*}{ EUSAAR2 } & 16.54 & & & 12.18 & \\
\hline & & 14.73 & & & 11.49 & \\
\hline & & 14.36 & & & 11.01 & \\
\hline & & 14.59 & & & 11.85 & \\
\hline & \multirow{4}{*}{ NIOSH870 } & 16.68 & 48.66 & 7.30 & 12.08 & 22.63 \\
\hline & & 16.80 & 51.10 & 7.45 & 12.35 & 23.61 \\
\hline & & 15.54 & 46.44 & 7.93 & 12.40 & 22.69 \\
\hline & & 16.69 & 49.49 & 7.51 & 12.34 & 23.12 \\
\hline \multirow{4}{*}{8} & \multirow{2}{*}{ EUSAAR2 } & 13.13 & 61.87 & 7.52 & 15.01 & 22.65 \\
\hline & & 16.25 & 70.48 & 7.02 & 12.89 & 25.48 \\
\hline & \multirow{2}{*}{ NIOSH870 } & 17.14 & 52.15 & 8.08 & 14.07 & 15.26 \\
\hline & & 16.90 & 60.79 & 7.34 & 13.91 & 23.15 \\
\hline
\end{tabular}


Table S8 (continued). OC (TOR) results per participant and protocol in $\mu \mathrm{g} / \mathrm{cm}^{2}$.

\begin{tabular}{|c|c|c|c|c|c|c|}
\hline Laboratory & OC & A & B & D & G & U \\
\hline \multirow{3}{*}{10} & \multirow{2}{*}{ EUSAAR2 } & 24.76 & 85.14 & 9.61 & 22.83 & 37.76 \\
\cline { 3 - 7 } & & 24.60 & 84.14 & 10.68 & 23.53 & 36.07 \\
\cline { 2 - 7 } & \multirow{2}{*}{ NIOSH-like } & 28.99 & 82.89 & 14.25 & 24.10 & 41.71 \\
\cline { 3 - 7 } & & 25.71 & 80.98 & 12.21 & 23.55 & 40.94 \\
\hline \multirow{3}{*}{$\mathbf{1 3}$} & \multirow{2}{*}{ EUSAAR2 } & 23.33 & 72.89 & 8.24 & 15.65 & 28.46 \\
\cline { 3 - 7 } & & 20.04 & 67.06 & 8.54 & 16.22 & 30.76 \\
\cline { 3 - 7 } & \multirow{2}{*}{ NIOSH870 } & 21.10 & 71.37 & 9.32 & 17.71 & 29.12 \\
\cline { 3 - 7 } & & 20.74 & 68.19 & 8.59 & 18.48 & 28.50 \\
\hline \multirow{3}{*}{$\mathbf{1 4}$} & \multirow{2}{*}{ EUSAAR2 } & 15.32 & 62.73 & 6.80 & 14.01 & 25.39 \\
\cline { 3 - 7 } & & 16.52 & 61.12 & 7.25 & 13.21 & 25.38 \\
\cline { 3 - 7 } & \multirow{2}{*}{ NIOSH870 } & 15.92 & 64.00 & 7.68 & 12.57 & 25.13 \\
\cline { 3 - 7 } & & 17.22 & 50.39 & 7.43 & 17.07 & 26.90 \\
\hline
\end{tabular}

Table S9. Normalized mean $(\mathrm{m})$, repeatability $\left(\mathrm{s}_{\mathrm{r}}\right)$ and reproducibility $\left(\mathrm{s}_{\mathrm{R}}\right)$ relative standard deviation, raw and corrected consensus values for TC per protocol.

\begin{tabular}{|c|c|c|c|c|c|}
\hline Protocol & Consensus values & m & $S_{r}$ & $S_{R}$ & Participants \\
\hline \multirow{2}{*}{ EUSAAR2 } & Raw & 1.064 & $\begin{array}{c}0.166 \\
(15.6 \%)\end{array}$ & $\begin{array}{c}0.212 \\
(19.9 \%)\end{array}$ & 17 \\
\hline & Corrected & 1.025 & $\begin{array}{c}0.117 \\
(11.4 \%)\end{array}$ & $\begin{array}{c}0.150 \\
(14.6 \%)\end{array}$ & 15 \\
\hline \multirow{2}{*}{ NIOSH870 } & Raw & 1.045 & $\begin{array}{c}0.134 \\
(12.9 \%)\end{array}$ & $\begin{array}{c}0.171 \\
(16.4 \%)\end{array}$ & 17 \\
\hline & Corrected & 1.015 & $\begin{array}{c}0.094 \\
(9.2 \%)\end{array}$ & $\begin{array}{c}0.119 \\
(11.7 \%)\end{array}$ & 15 \\
\hline
\end{tabular}

Table S10. Normalized mean $(m)$, repeatability $\left(\mathrm{s}_{\mathrm{r}}\right)$ and reproducibility relative standard deviation (SR), raw and corrected consensus values for $\mathrm{EC}$ per protocol.

\begin{tabular}{llcccc}
\hline \multirow{2}{*}{ Protocol } & Consensus values & $\mathbf{m}$ & $\mathbf{S}_{\mathbf{r}}$ & $\mathrm{S}_{\mathbf{R}}$ & Participants \\
\hline \multirow{2}{*}{ EUSAAR2 } & Raw & 1.025 & $\begin{array}{c}0.238 \\
(23.2 \%)\end{array}$ & $\begin{array}{c}0.303 \\
(29.5 \%)\end{array}$ & 17 \\
\cline { 2 - 6 } & Corrected & $\mathbf{1 . 0 5 1}$ & $\begin{array}{c}\mathbf{0 . 1 6 1} \\
(\mathbf{1 5 . 3 \% )}\end{array}$ & $\begin{array}{c}\mathbf{0 . 2 0 5} \\
(\mathbf{1 9 . 5 \% )}\end{array}$ & $\mathbf{1 5}$ \\
\hline \multirow{2}{*}{ NIOSH870 } & Raw & 1.064 & $\begin{array}{c}0.276 \\
(25.9 \%)\end{array}$ & $\begin{array}{c}0.353 \\
(33.1 \%)\end{array}$ & 17 \\
\cline { 2 - 6 } & Corrected & $\mathbf{1 . 0 1 3}$ & $\begin{array}{c}\mathbf{0 . 2 0 2} \\
(\mathbf{1 9 . 9 \% )}\end{array}$ & $\begin{array}{c}\mathbf{0 . 2 5 8} \\
(\mathbf{2 5 . 5 \% )}\end{array}$ & $\mathbf{1 5}$ \\
\hline
\end{tabular}


Table S11. Overview of tests on filter load homogeneity by replicate analysis on two HVS quartz filters (Pall Tissuquartz and Whatman QMA). OC, EC, TC, and EC/TC

concentrations and relative standard deviation reported per filter.

\begin{tabular}{|c|c|c|c|c|}
\hline & OC & EC & TC & EC/TC \\
\hline Average filter $1\left(\mu \mathrm{g} / \mathrm{cm}^{2}\right)^{a}$ & 26.66 & 12.18 & 38.83 & 0.31 \\
\hline RSD $\%$ filter $\mathbf{1}^{\mathrm{a}}$ & 6.4 & 10.6 & 5.3 & 8.7 \\
\hline Average filter $2\left(\mu \mathrm{g} / \mathrm{cm}^{2}\right)^{b}$ & 19.54 & 7.01 & 26.54 & 0.26 \\
\hline RSD $\%$ filter $2^{b}$ & 8.7 & 9.9 & 6.3 & 11.3 \\
\hline
\end{tabular}




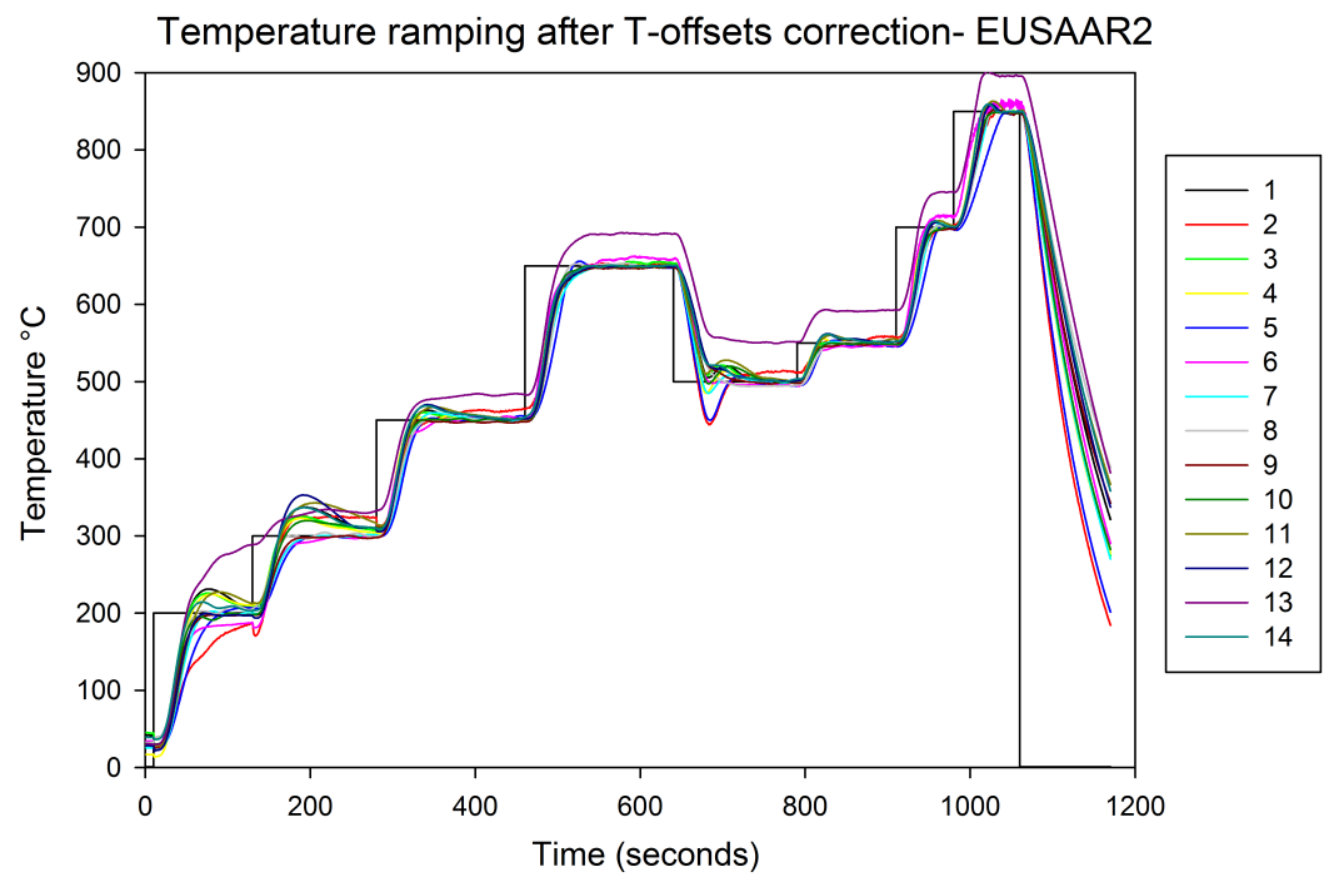

Figure S1. Heating profile per participant during analysis with EUSAAR2 thermal protocol after temperature offsets correction.

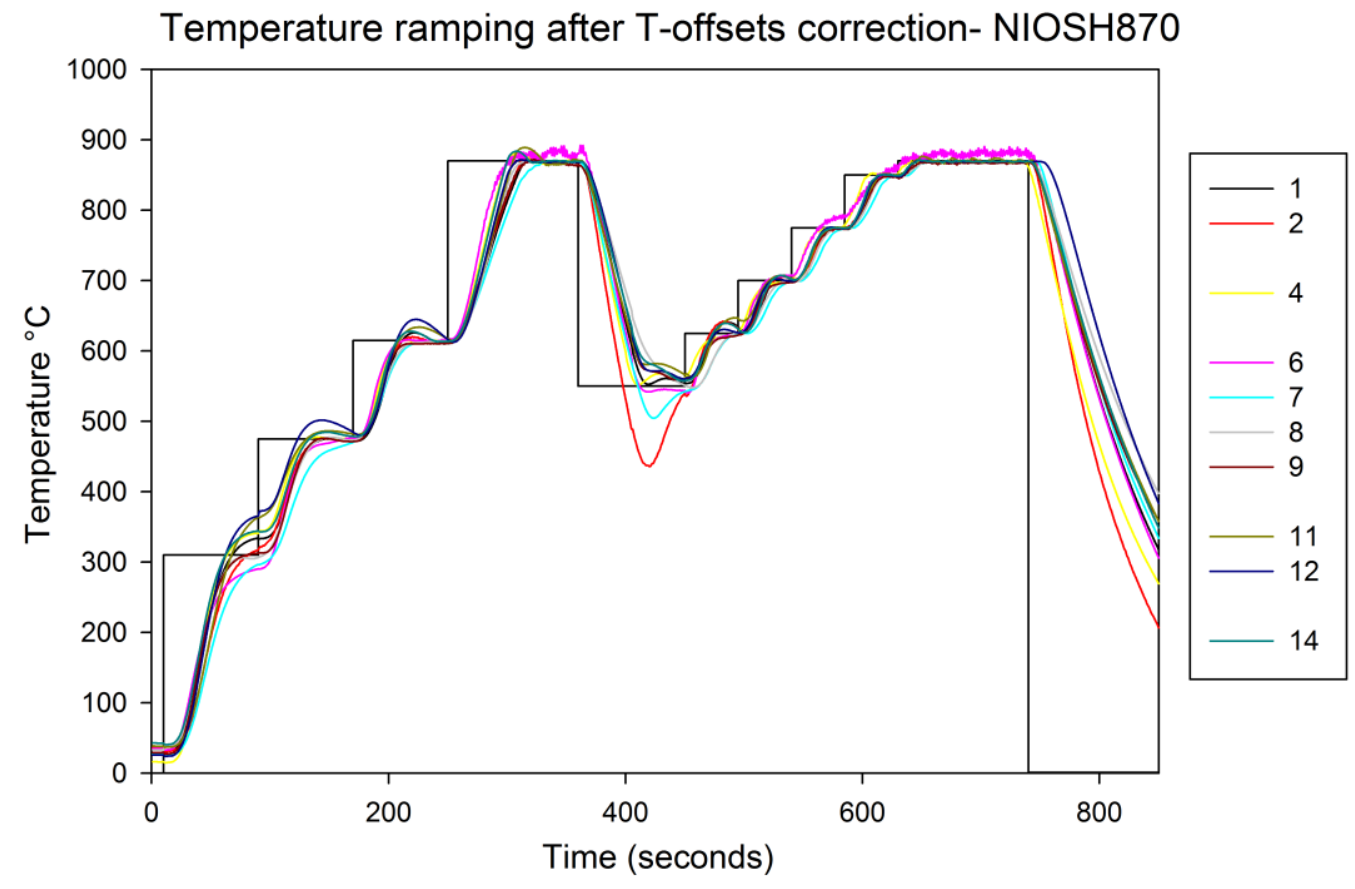

Figure S2. Heating profile per participant during analysis with NIOSH870 thermal protocol after temperature offsets correction. 


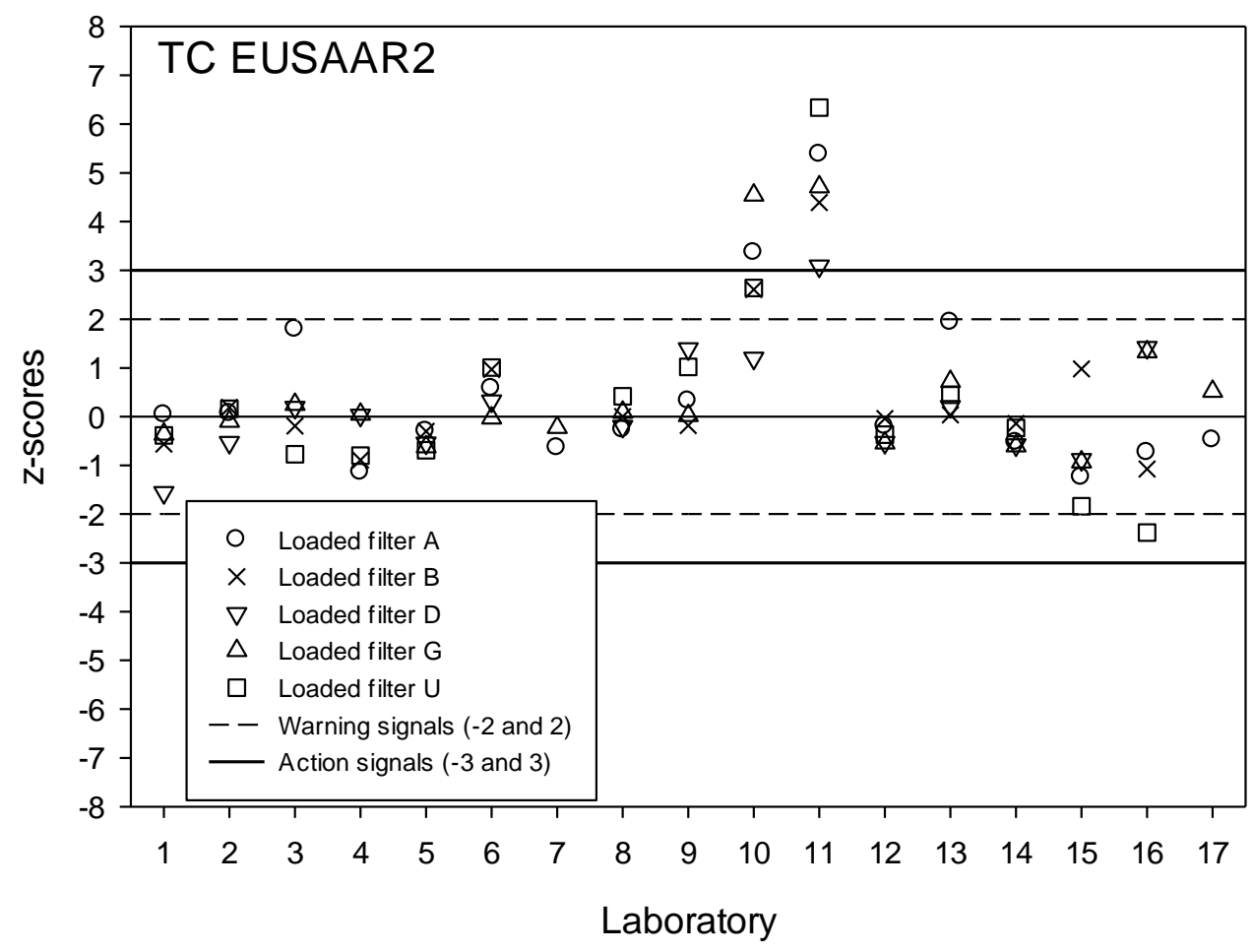

Figure S3. z-scores for TC by EUSAAR2 protocol per participant and filter, calculated from robust means and fit for purpose relative standard deviation of $8.3 \%$.

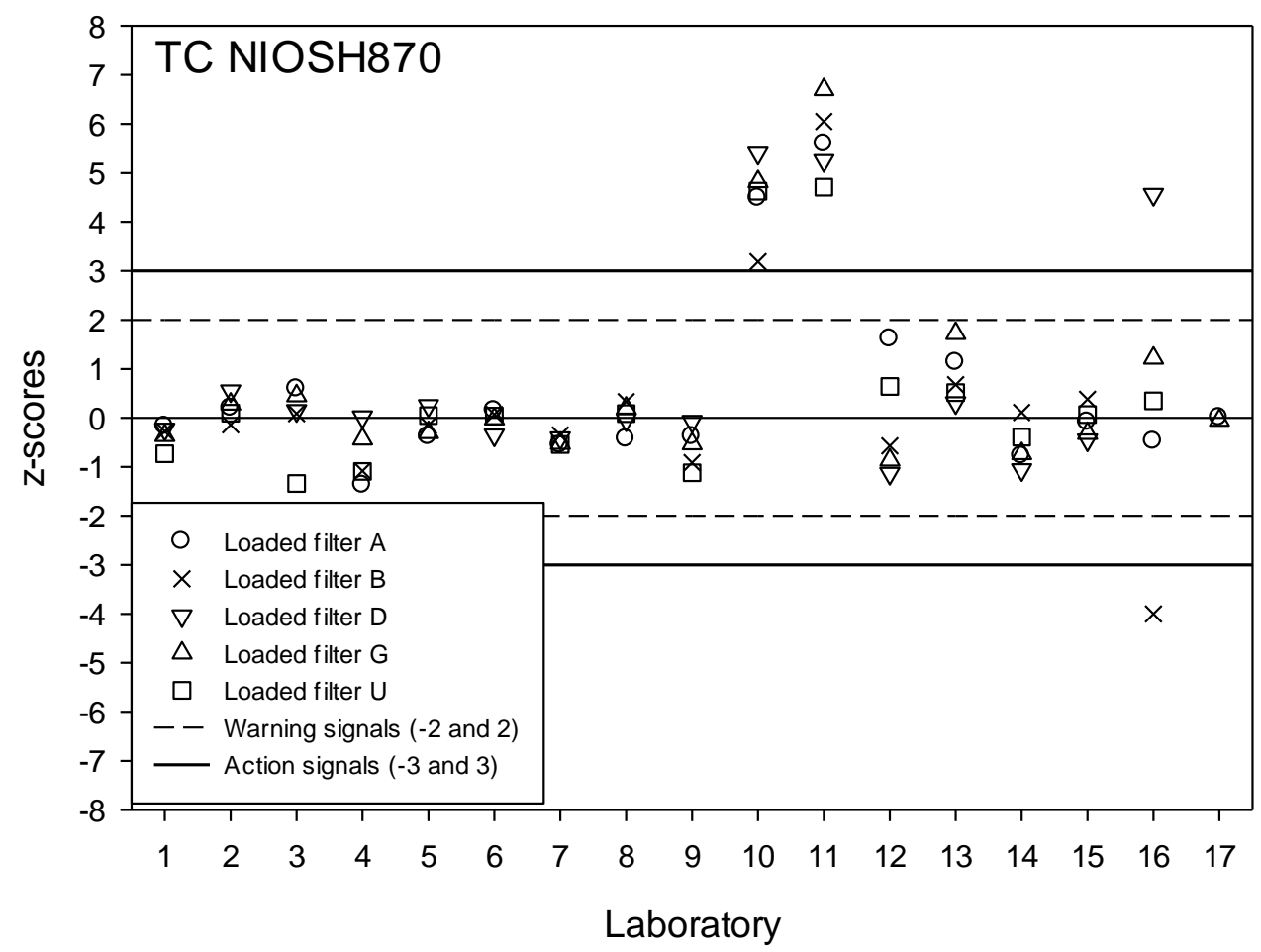

Figure S4. z-scores for TC by NIOSH870 protocol per participant and filter, calculated from the robust means and fit for purpose relative standard deviation of $8.3 \%$. 


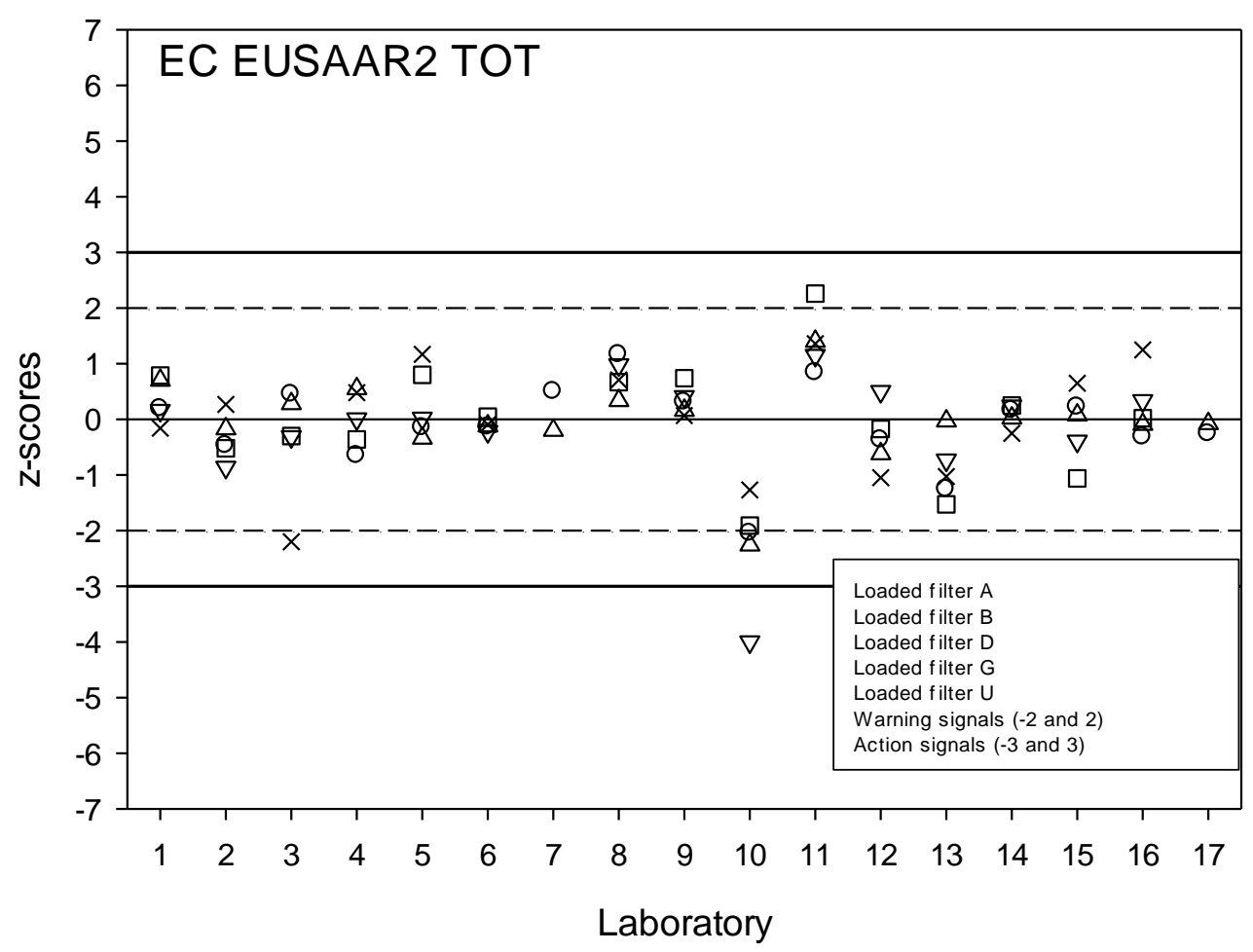

Figure S5. z-scores for EC for the EUSAAR2 protocol on TOT mode, per participant and filter, calculated from the robust means and fit for purpose relative standard deviation of $25 \%$.

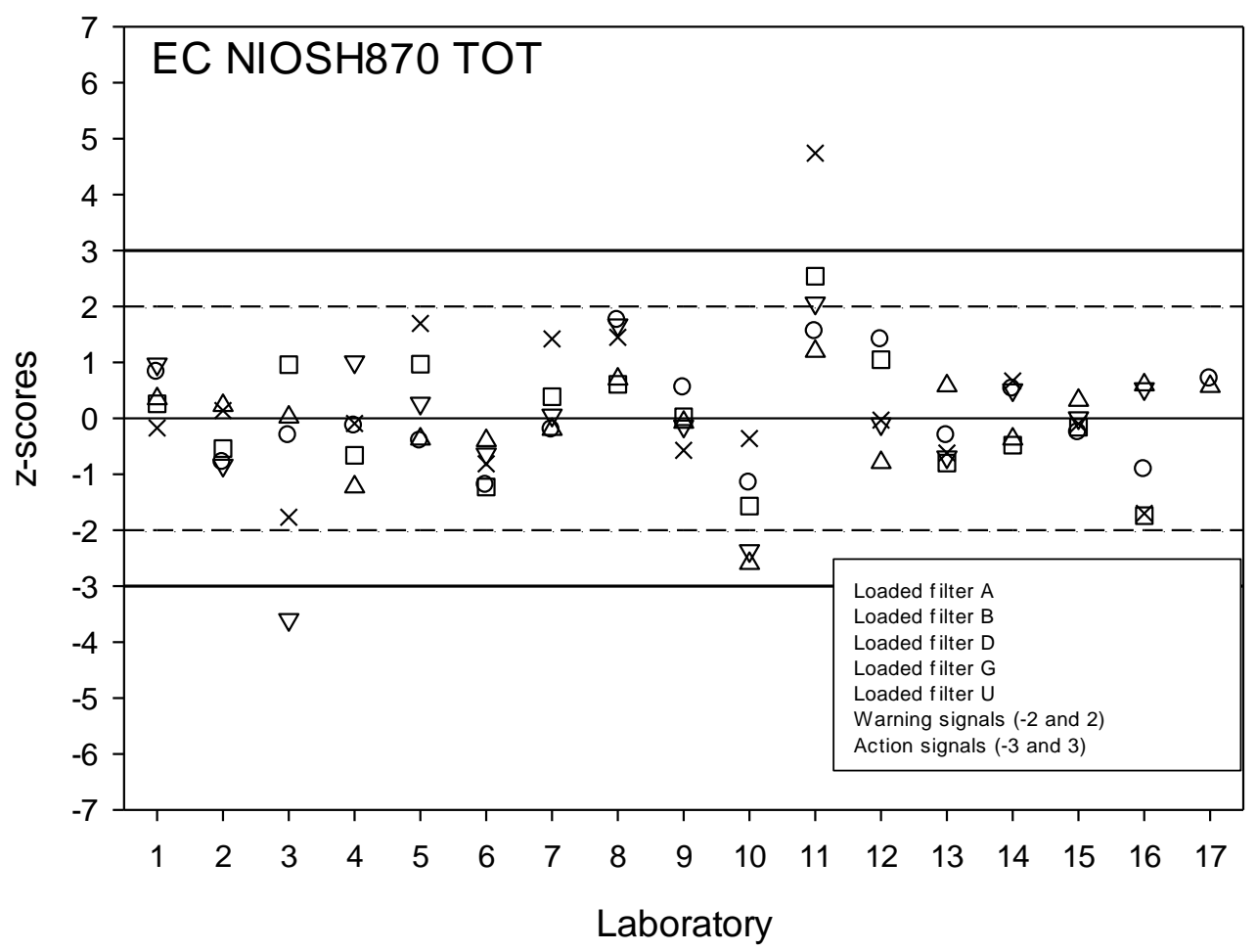


Figure S6. z-scores for EC for the NIOSH870 protocol on TOT mode, per participant and filter, calculated from the robust means and fit for purpose relative standard deviation of $25 \%$.

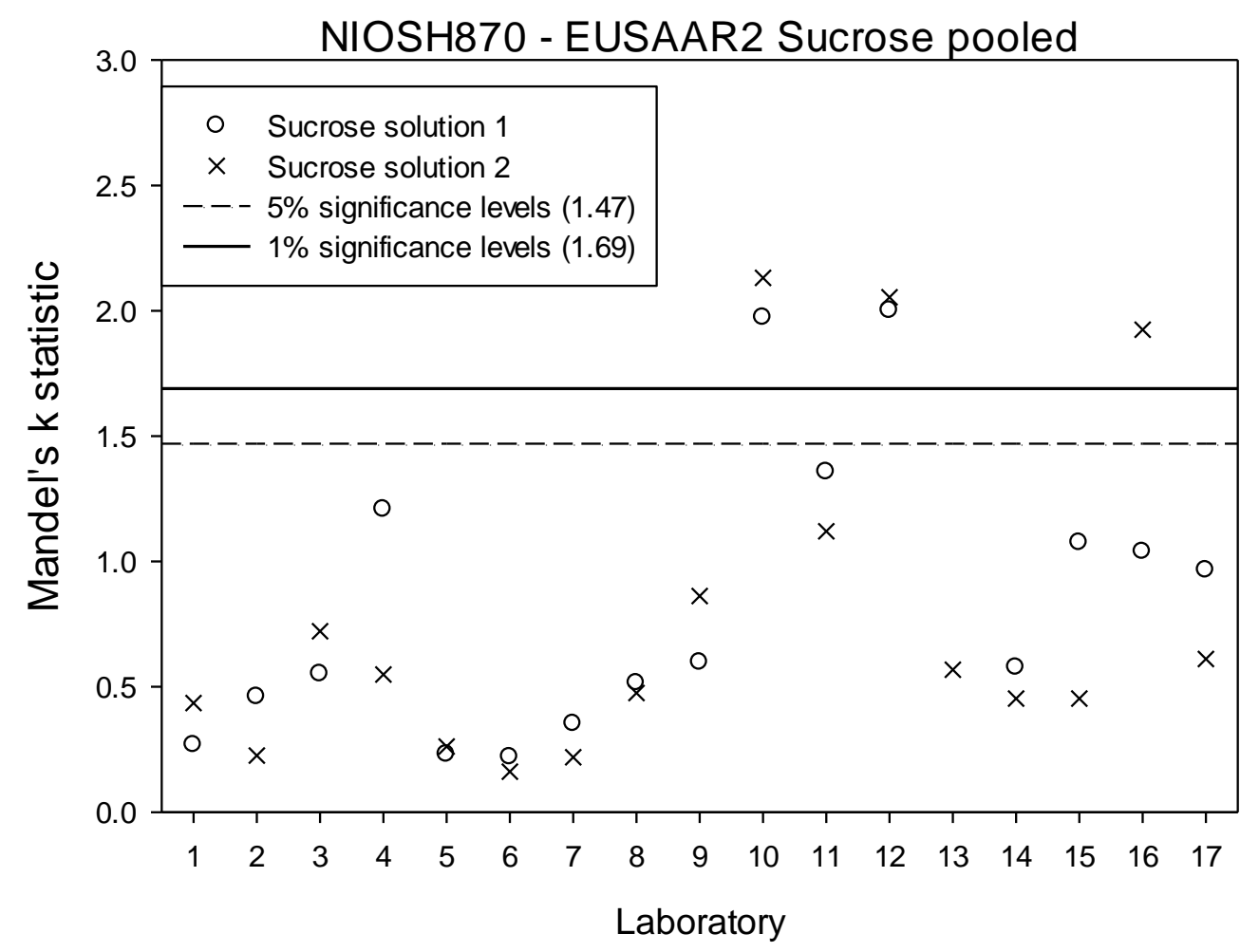

Figure S7. Mandel's k statistic values (within laboratory consistency) calculated for the pooled TC results, EUSAAR2 and NIOSH870, per participant and sucrose solution. 


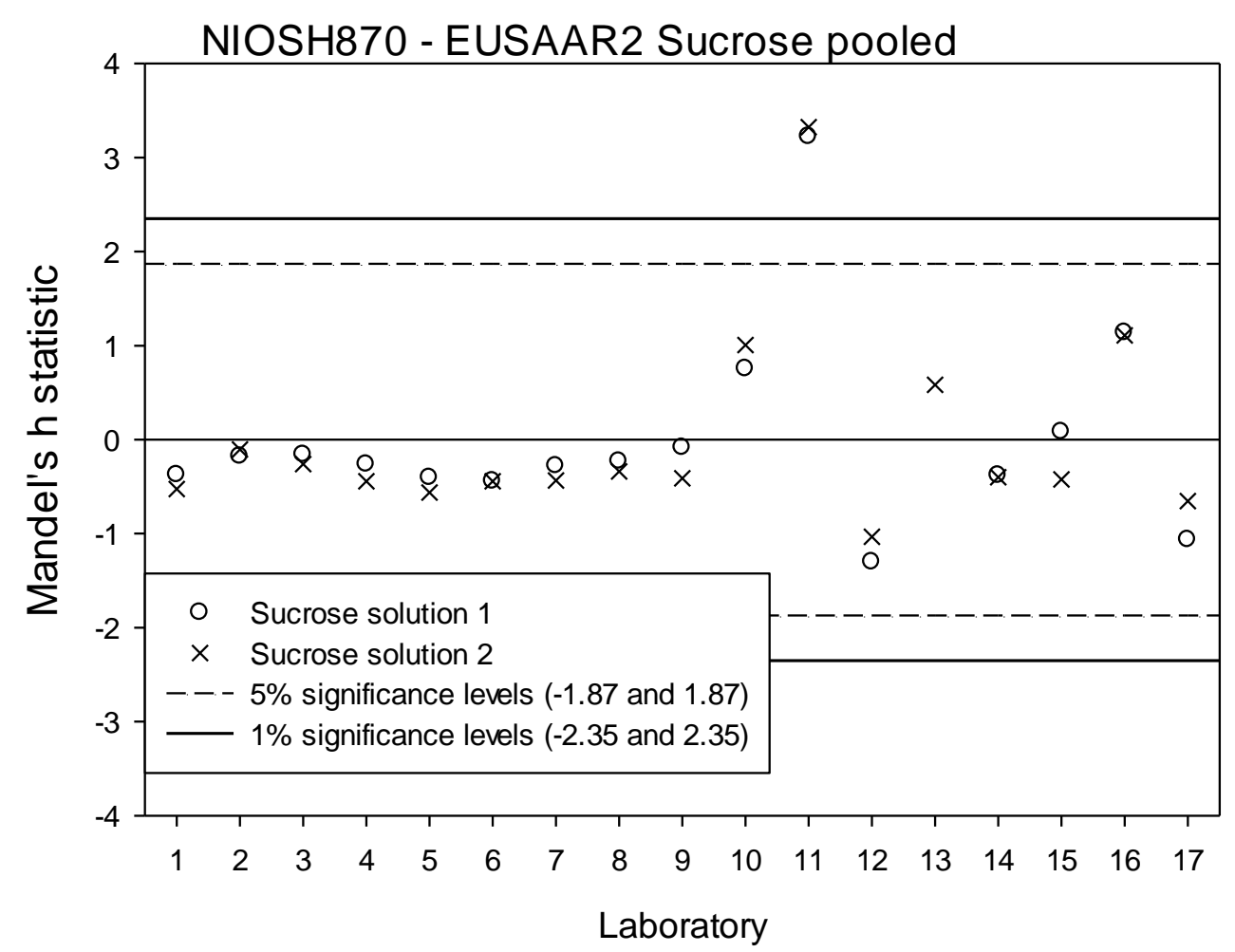

Figure S8. Mandel's h statistic values (between laboratory consistency) calculated for the pooled TC results, EUSAAR2 and NIOSH870, per participant and sucrose solution.

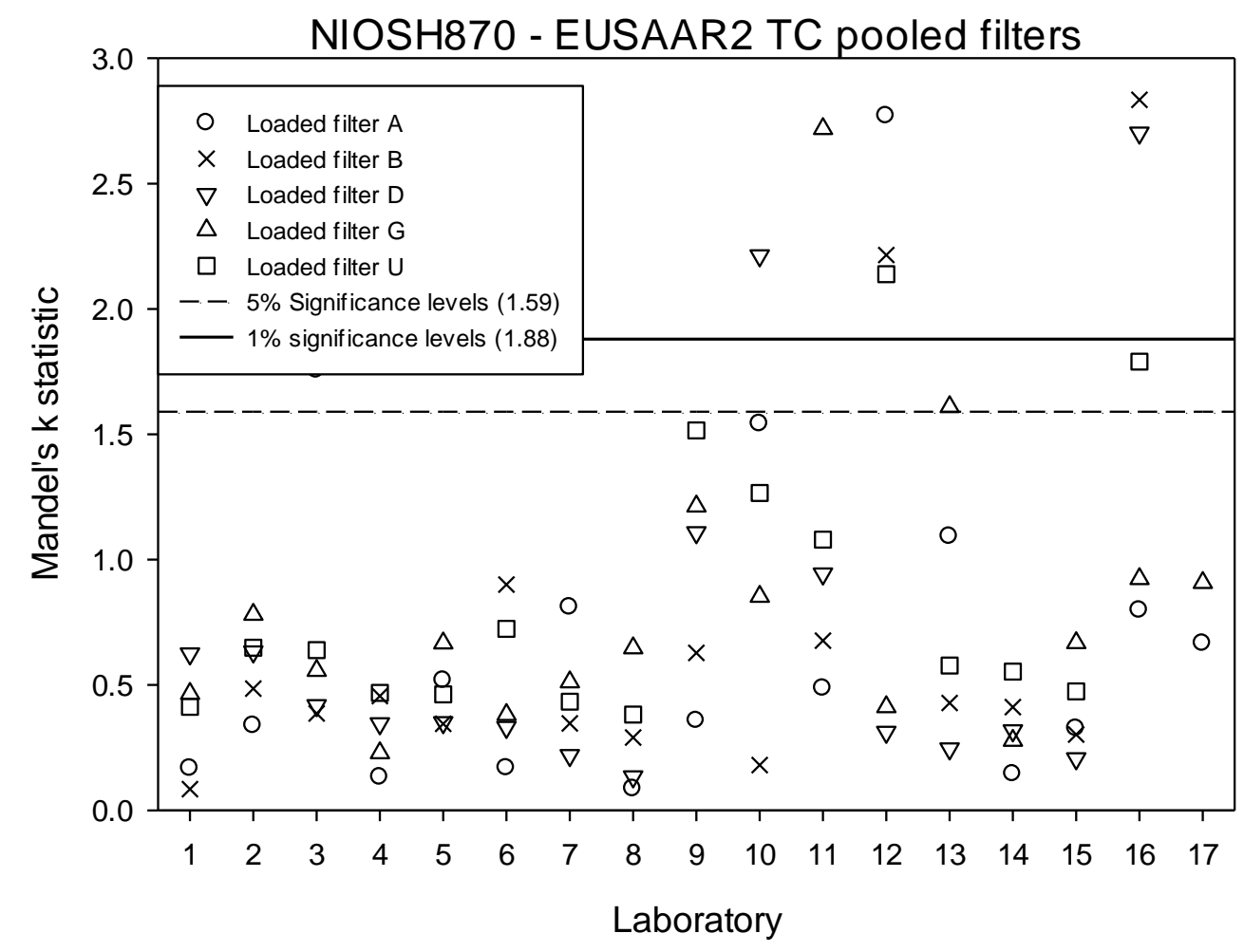

Figure S9. Mandel's k statistic values (within laboratory consistency) calculated for the pooled TC results, EUSAAR2 and NIOSH870, per participant and filter. 


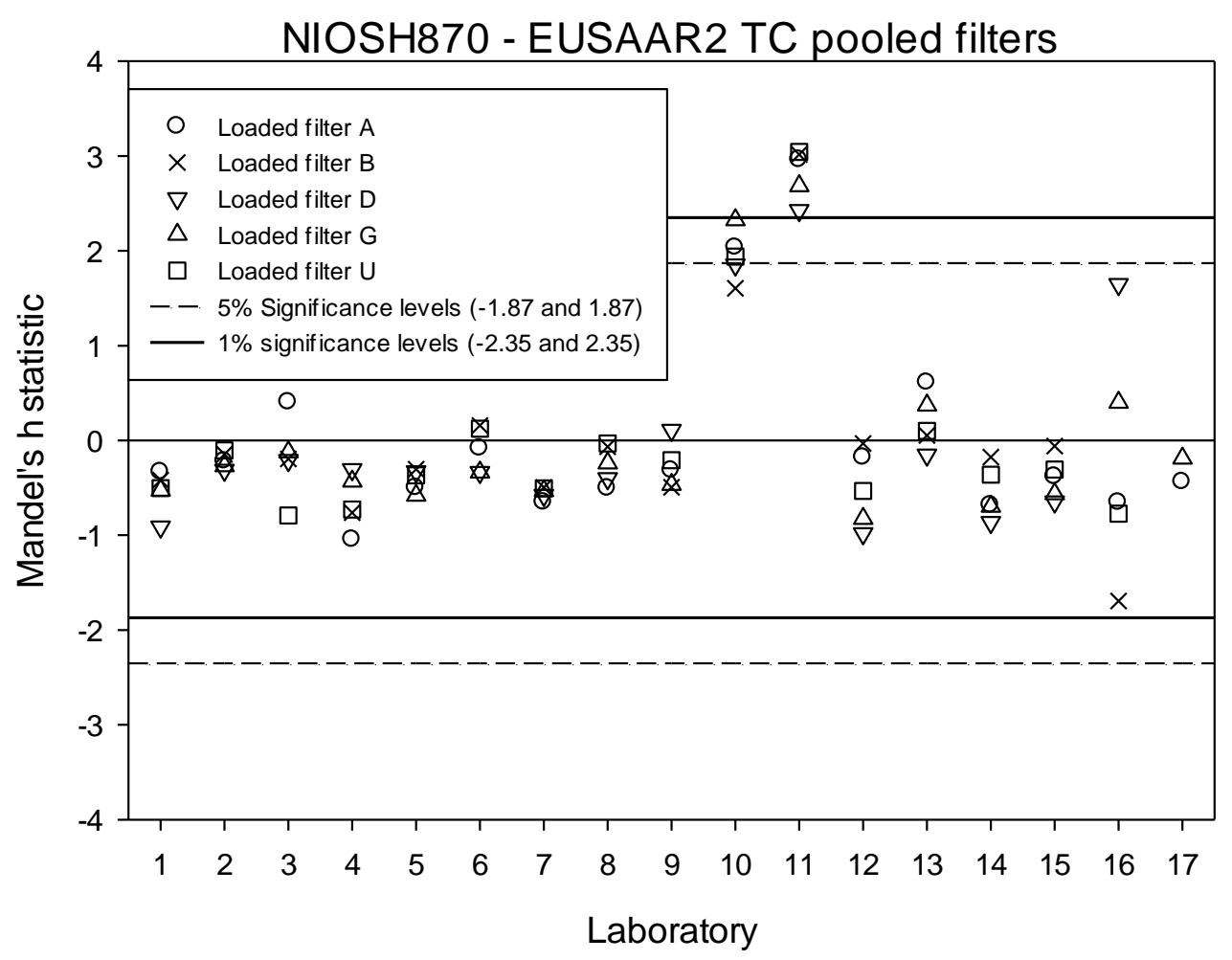

Figure S10. Mandel's h statistic values (between laboratory consistency) calculated for the pooled TC results, EUSAAR2 and NIOSH870, per participant and filter.

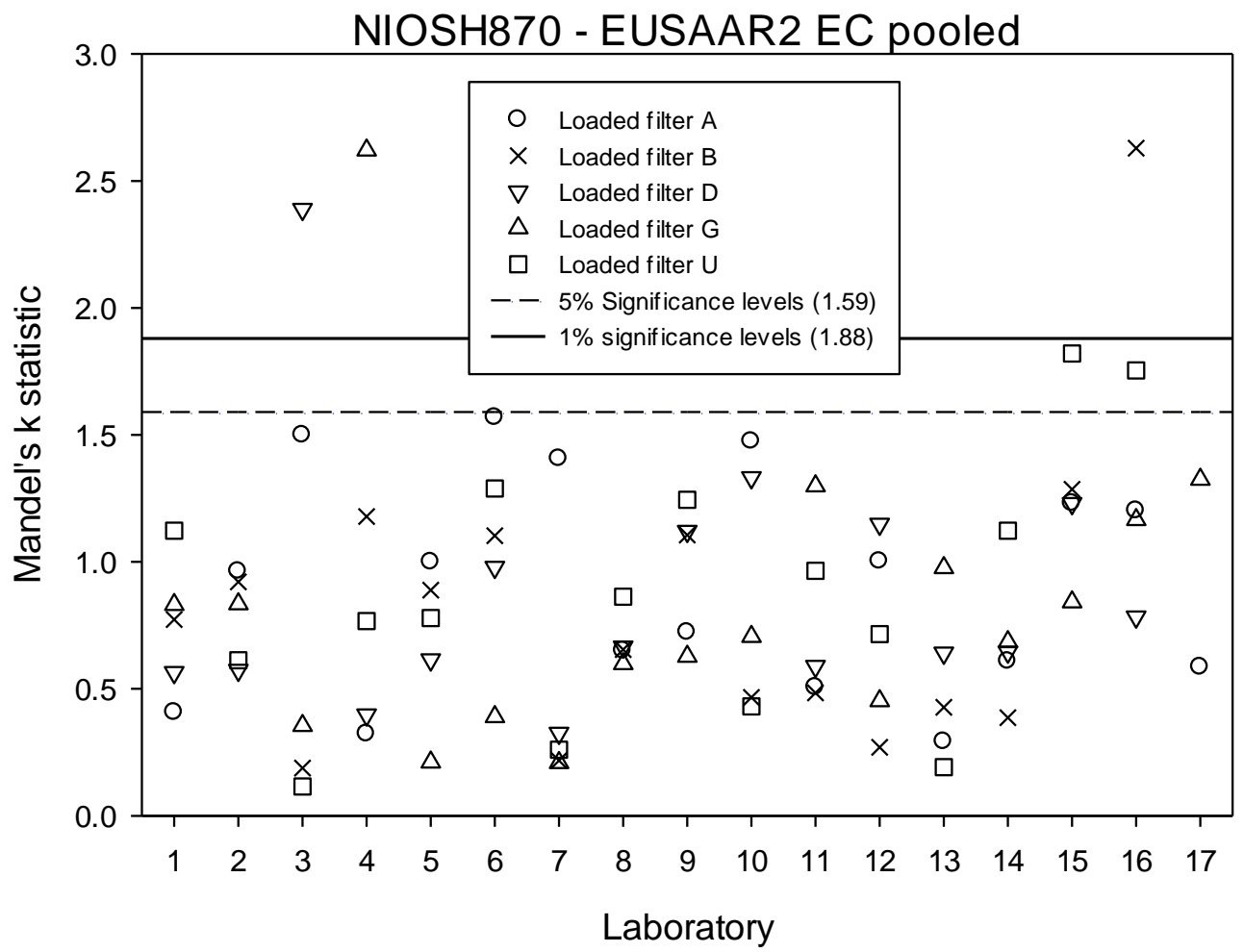

Figure S11. Mandel's k statistic values (within laboratory consistency) calculated for the pooled EC (TOT) EUSAAR2 and NIOSH870 results per participant and filter. 


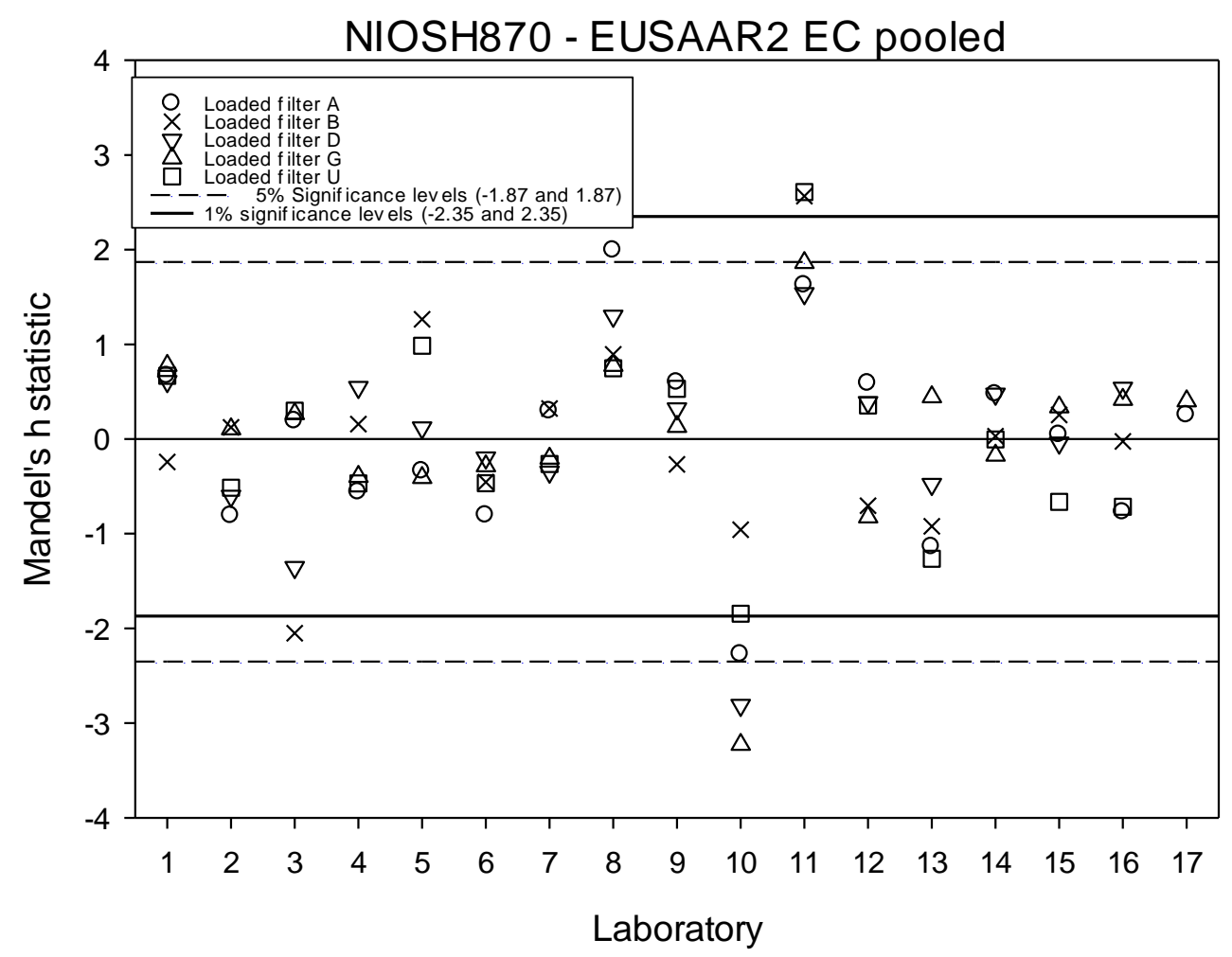

Figure S12. Mandel's h statistic values (between laboratory consistency) calculated for the pooled EC (TOT) EUSAAR2 and NIOSH870 results per participant and filter.
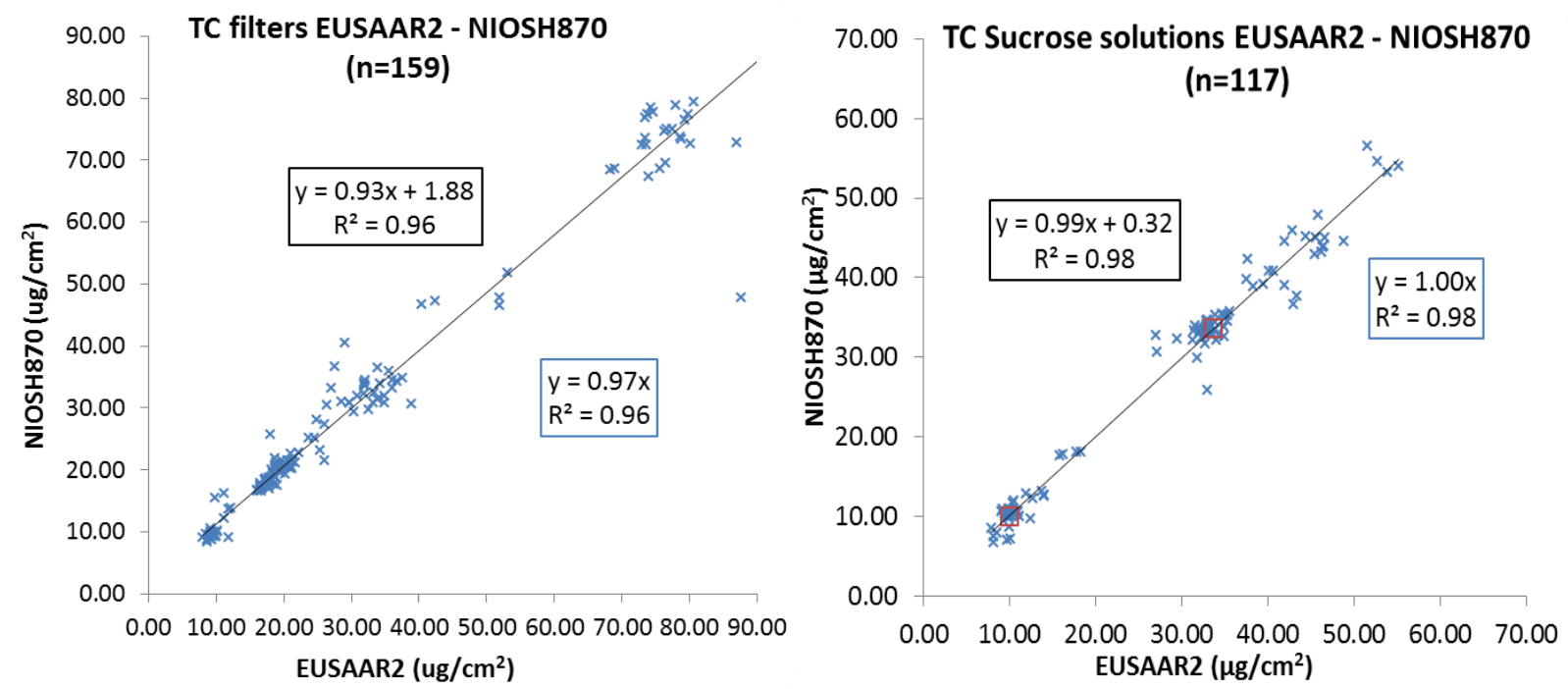

Figure 113. Comparison of pooled raw TC results between EUSAAR2 and NIOSH870 for filter samples and sucrose solutions. The red squares indicate the actual concentration of the 2 sucrose solutions. 

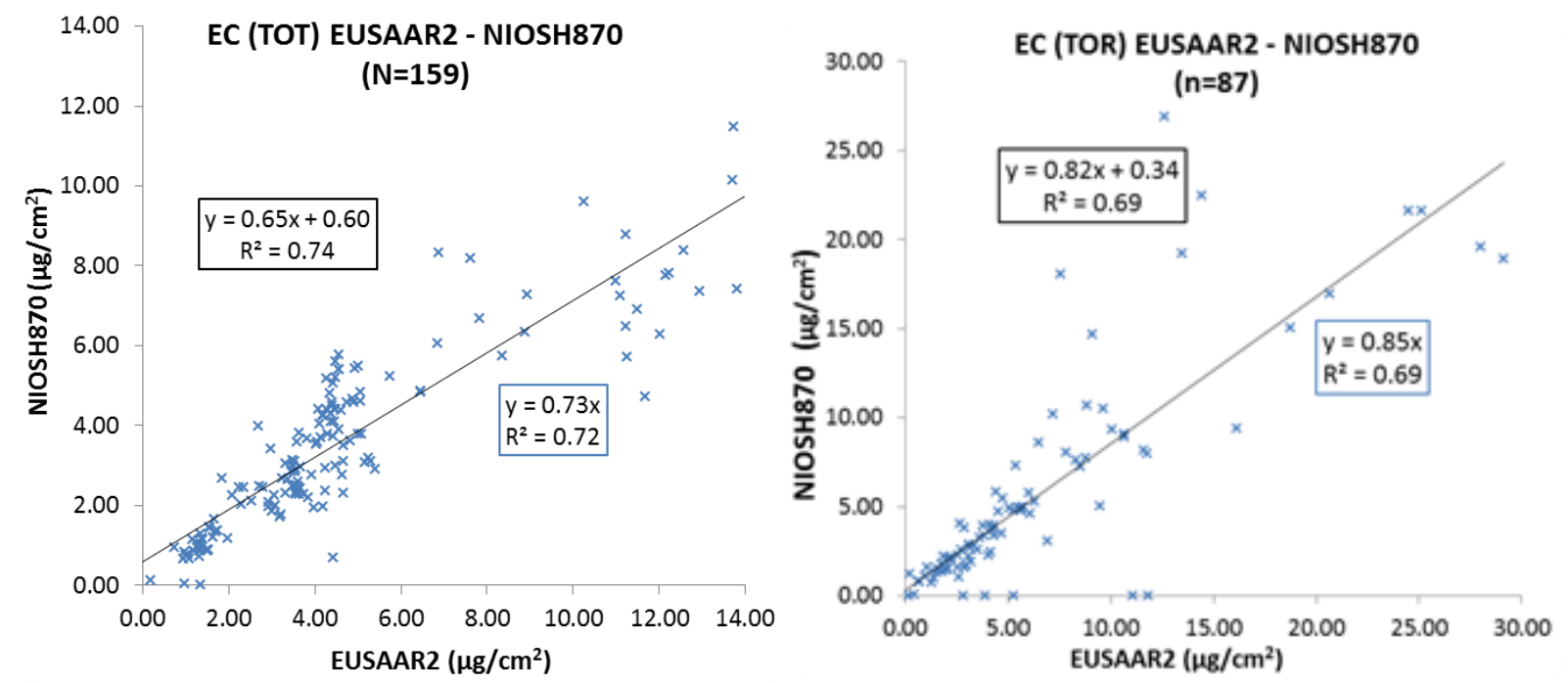

Figure S14. Comparison of pooled EC raw results between EUSAAR2 and NIOSH870 protocols with the use of transmittance (TOT) or reflectance (TOR).
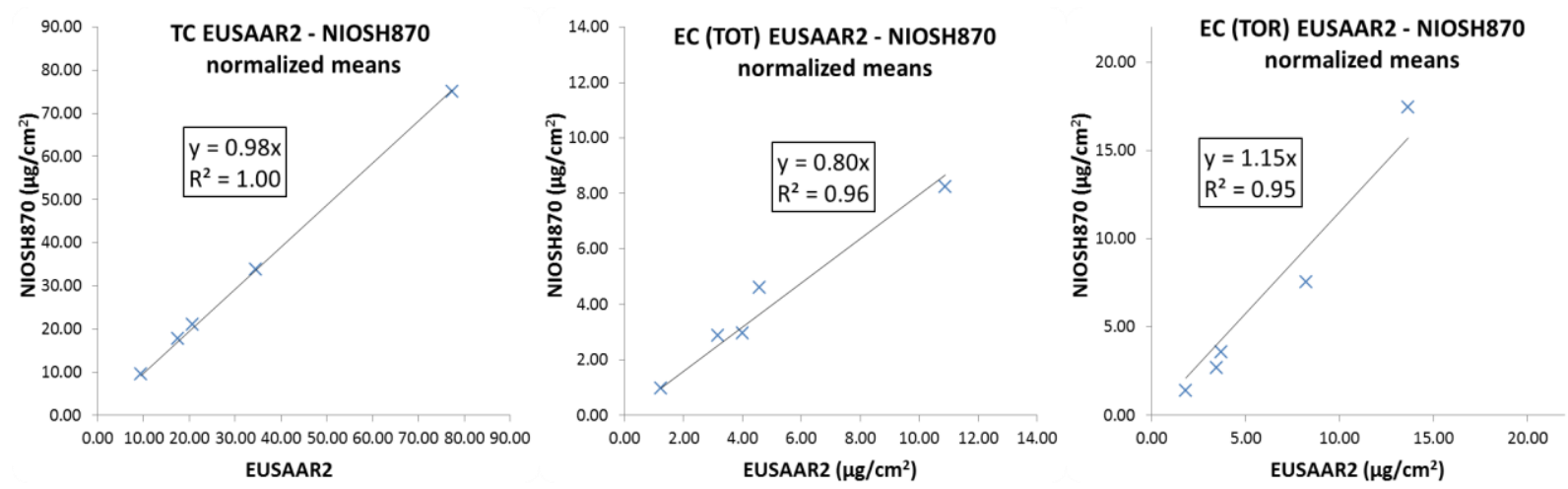

Figure S15. Comparison of pooled TC (TOT) and EC (TOT and TOR) normalized results between EUSAAR2 and NIOSH870 protocols. 


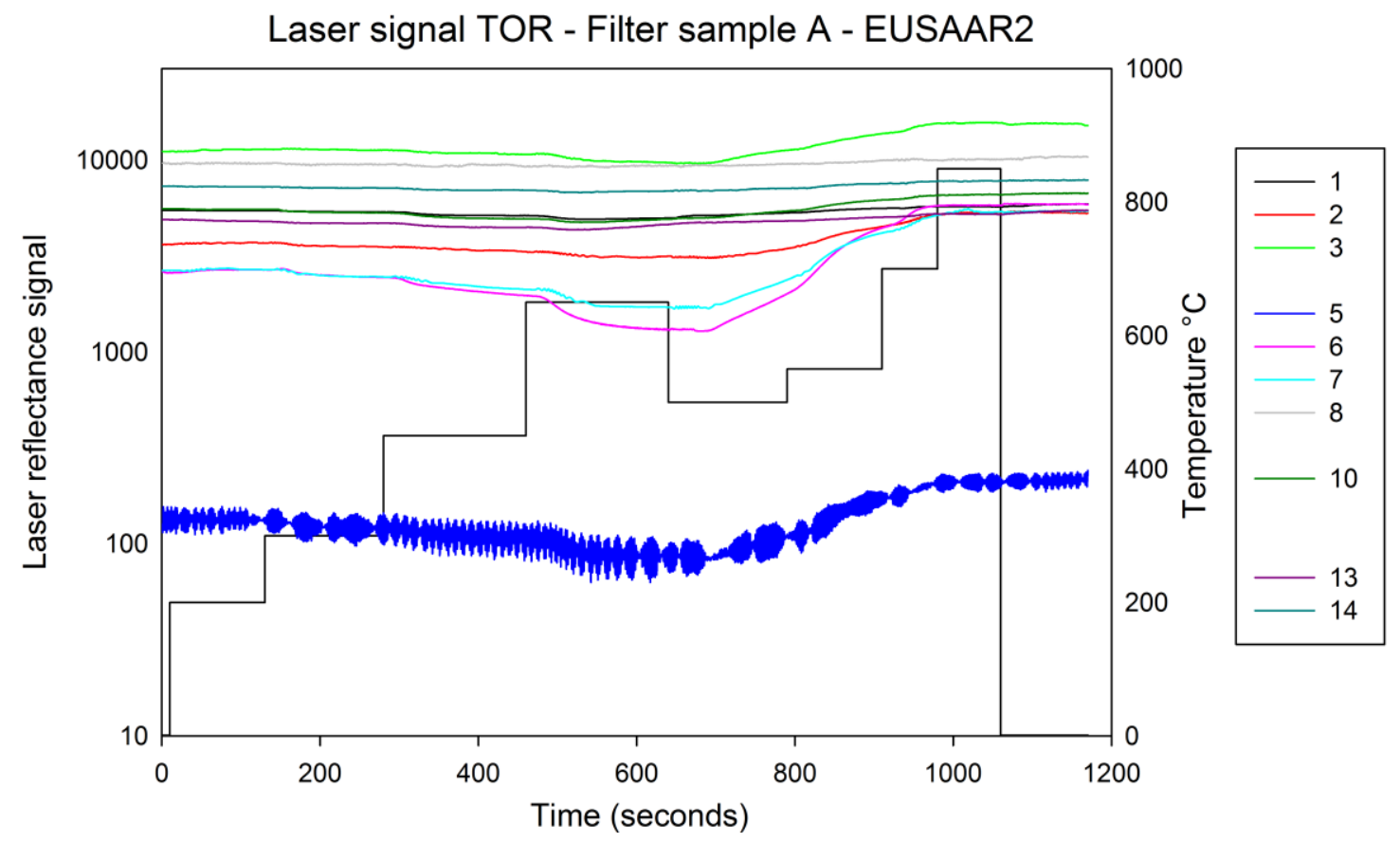

Figure 216. Laser reflectance signal during filter sample A analysis with the use of the EUSAAR2 thermal protocol.

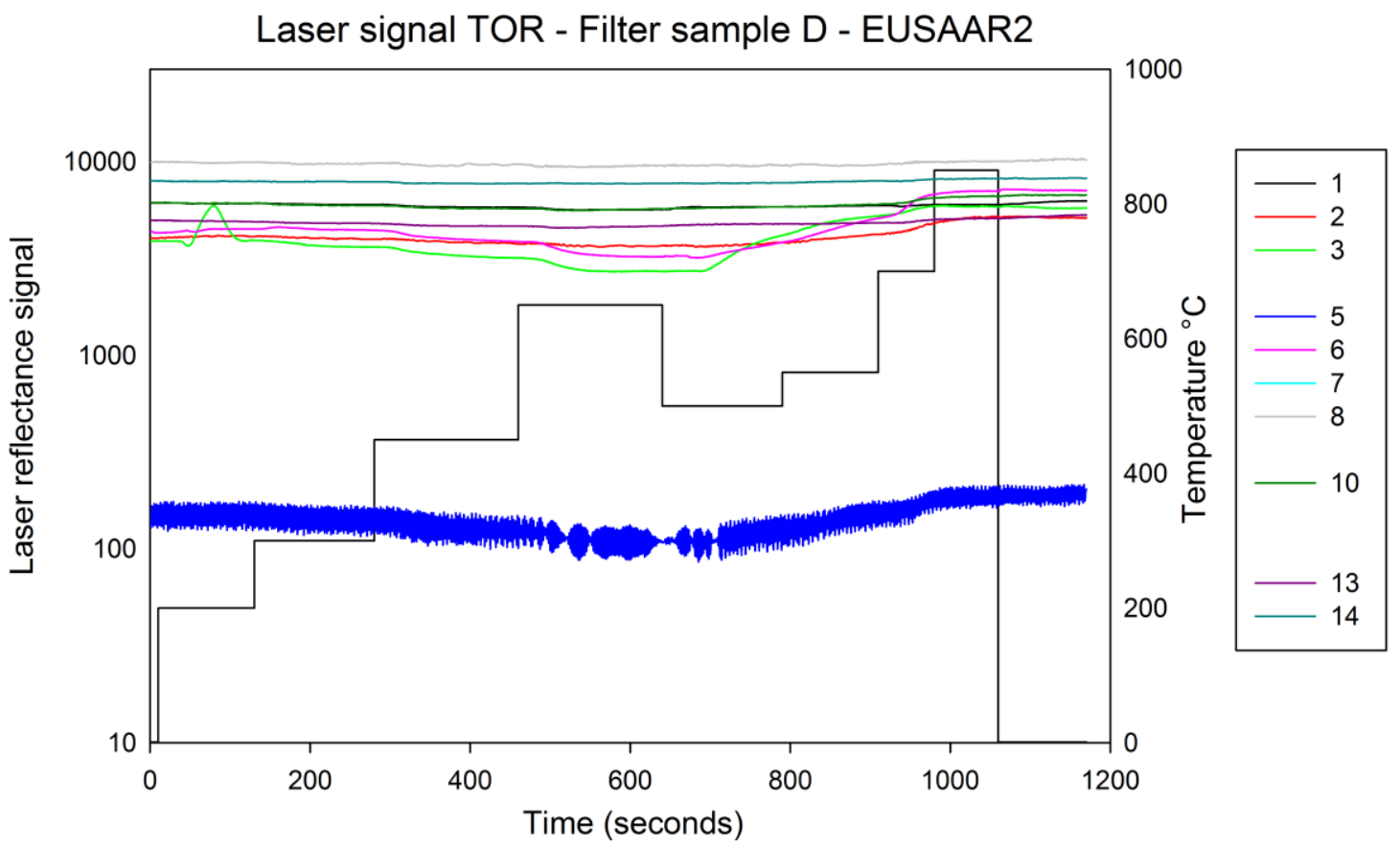

Figure S17. Laser reflectance signal during filter sample D analysis with the use of the EUSAAR2 thermal protocol. 


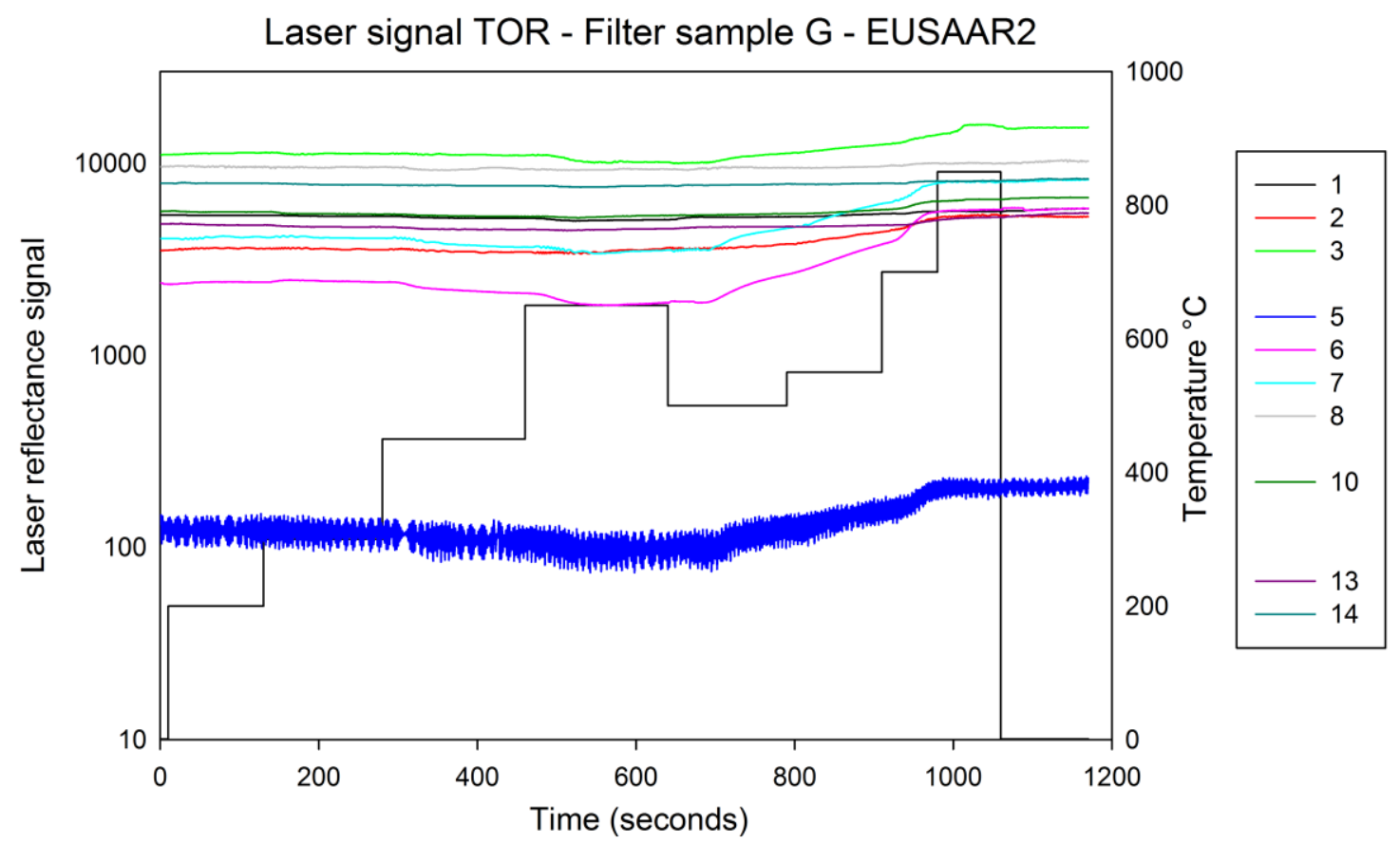

Figure S18. Laser reflectance signal during filter sample $G$ analysis with the use of the EUSAAR2 thermal protocol.

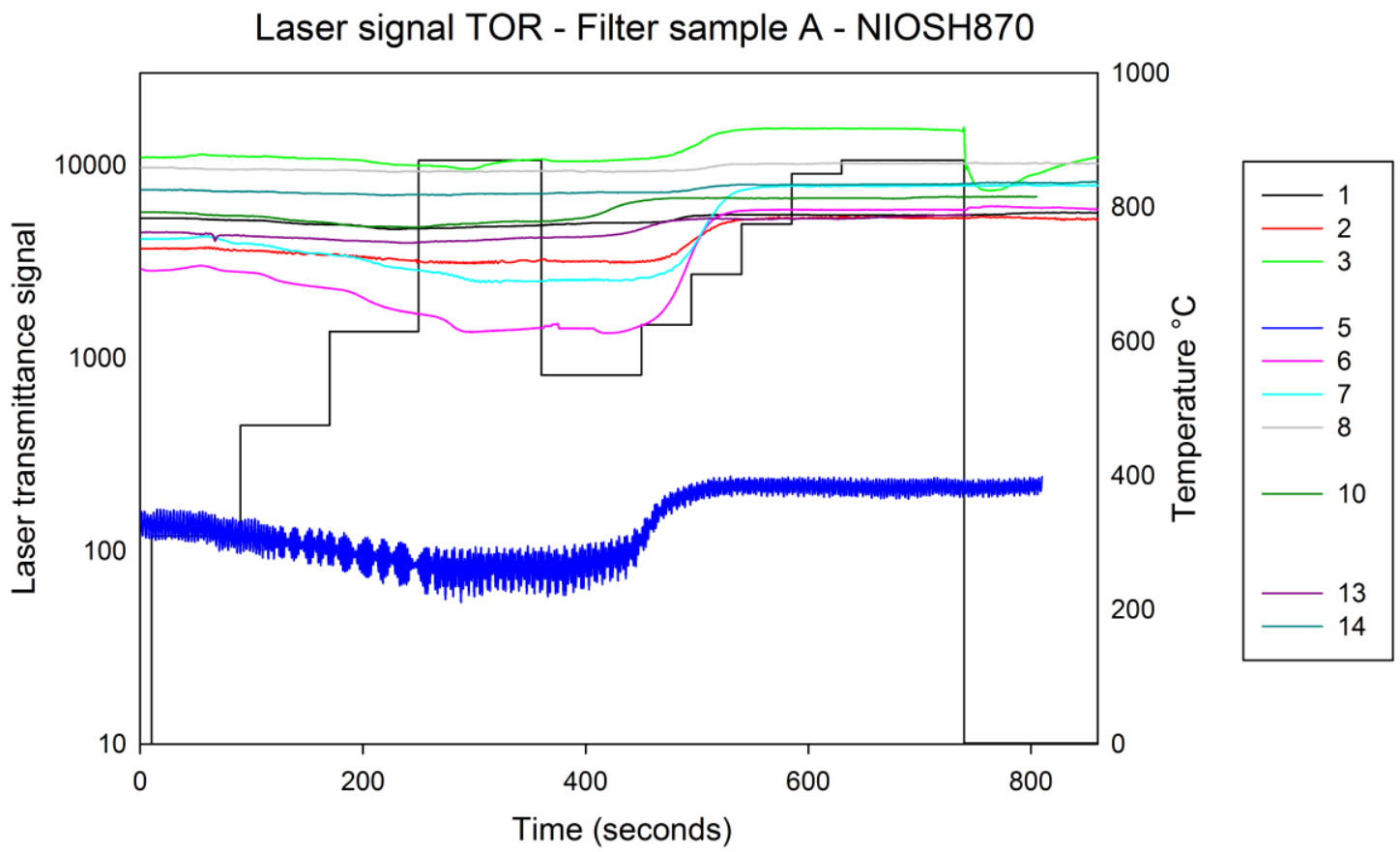

Figure S19. Laser reflectance signal during filter sample A analysis with the use of the NIOSH870 thermal protocol. 


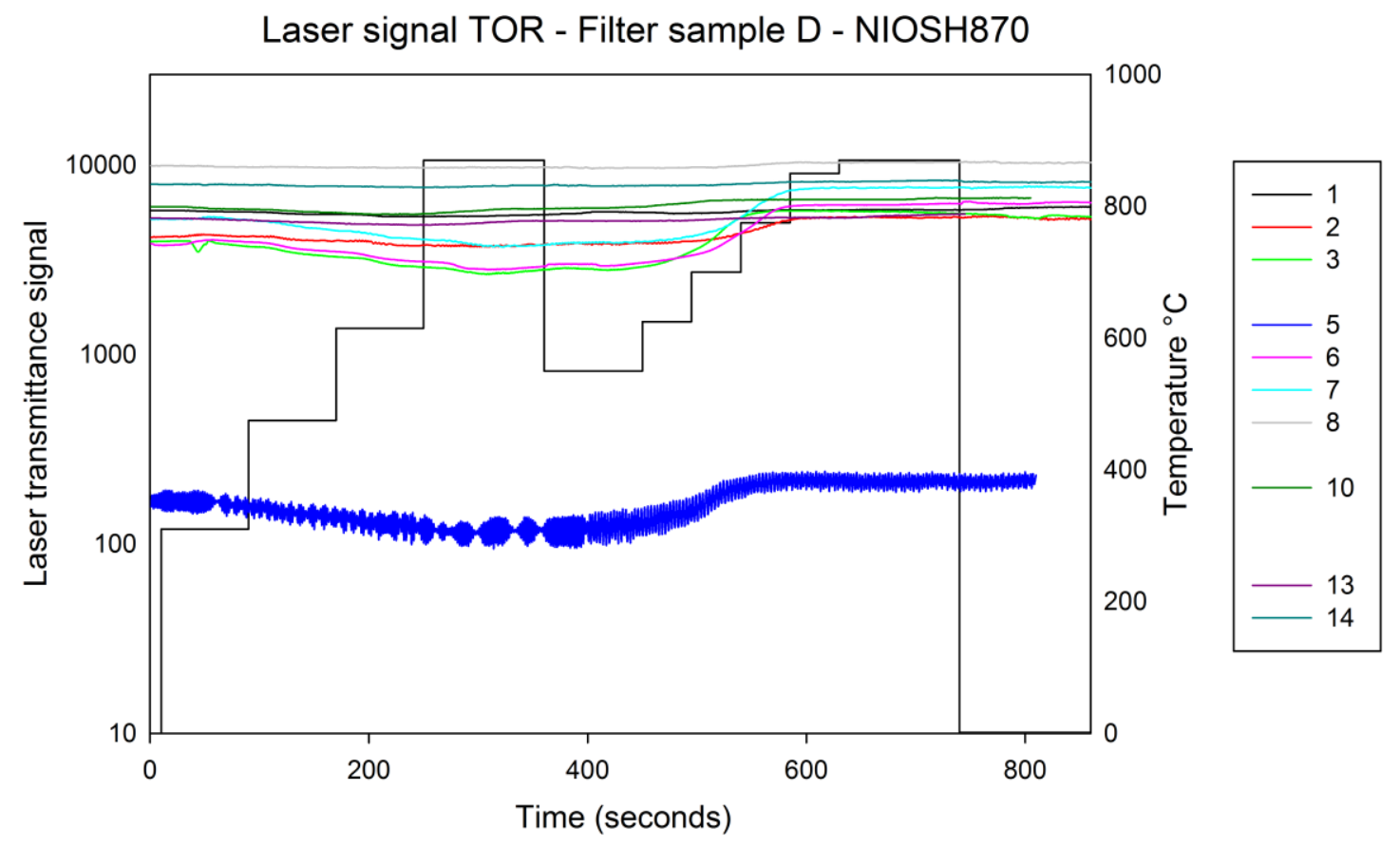

Figure S20. Laser reflectance signal during filter sample D analysis with the use of the NIOSH870 thermal protocol.

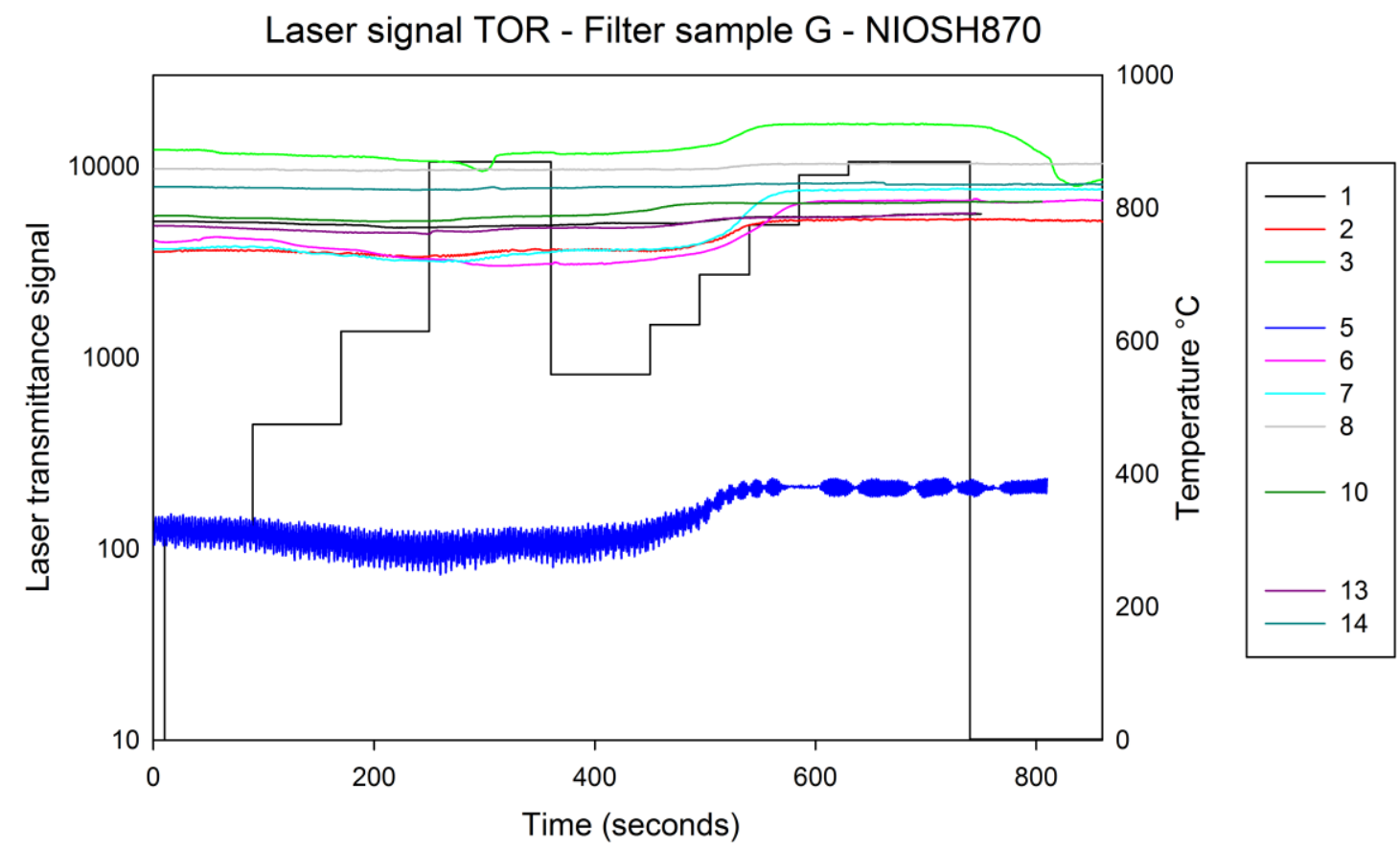

Figure S21. Laser reflectance signal during filter sample $G$ analysis with the use of the NIOSH870 thermal protocol. 


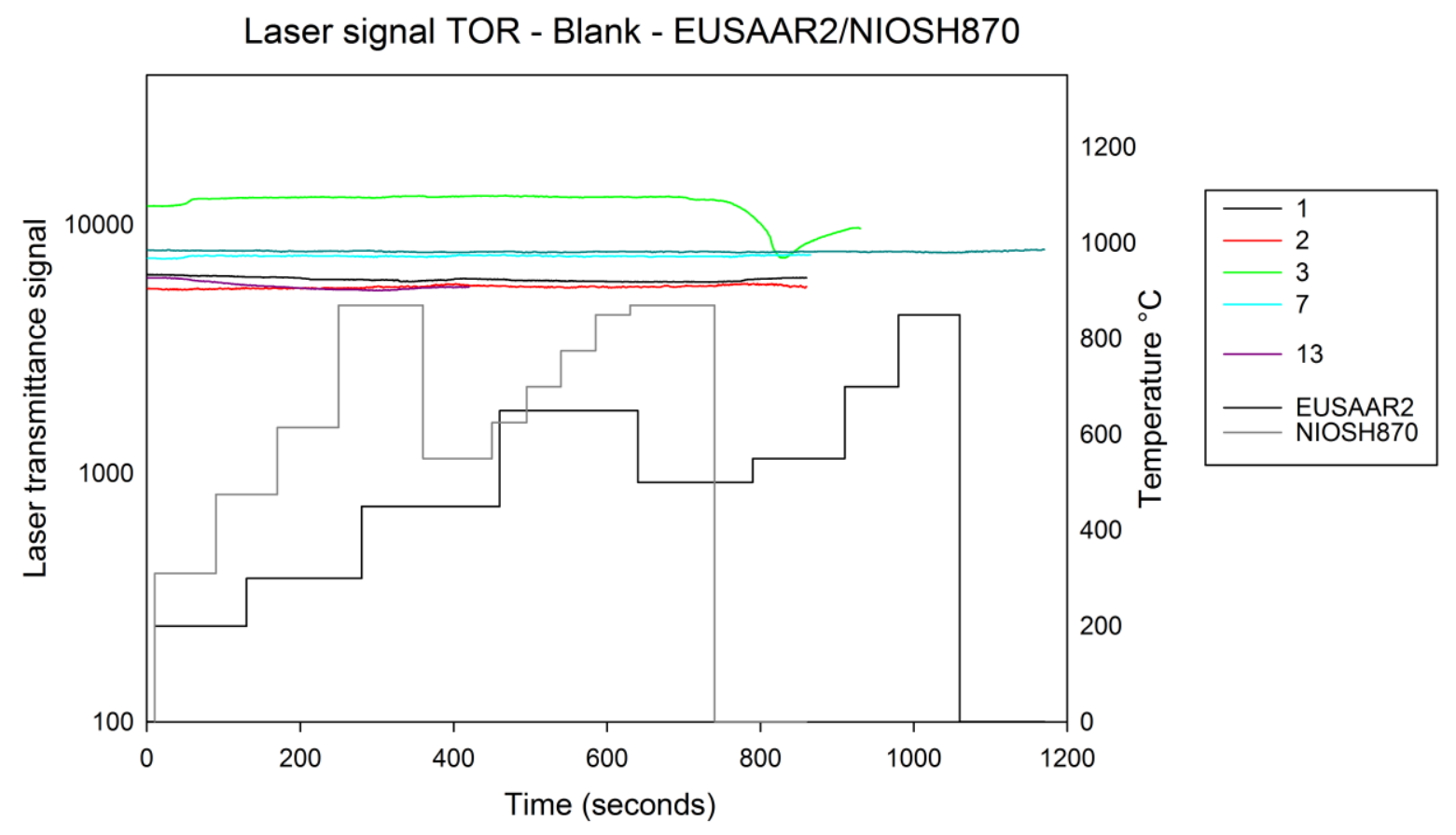

Figure S22. Laser reflectance signal during instrument blank filter analysis with the use of the EUSAAR2 or NIOSH870 thermal protocol. (Participant 13 used a shorter protocol for the specific analysis).

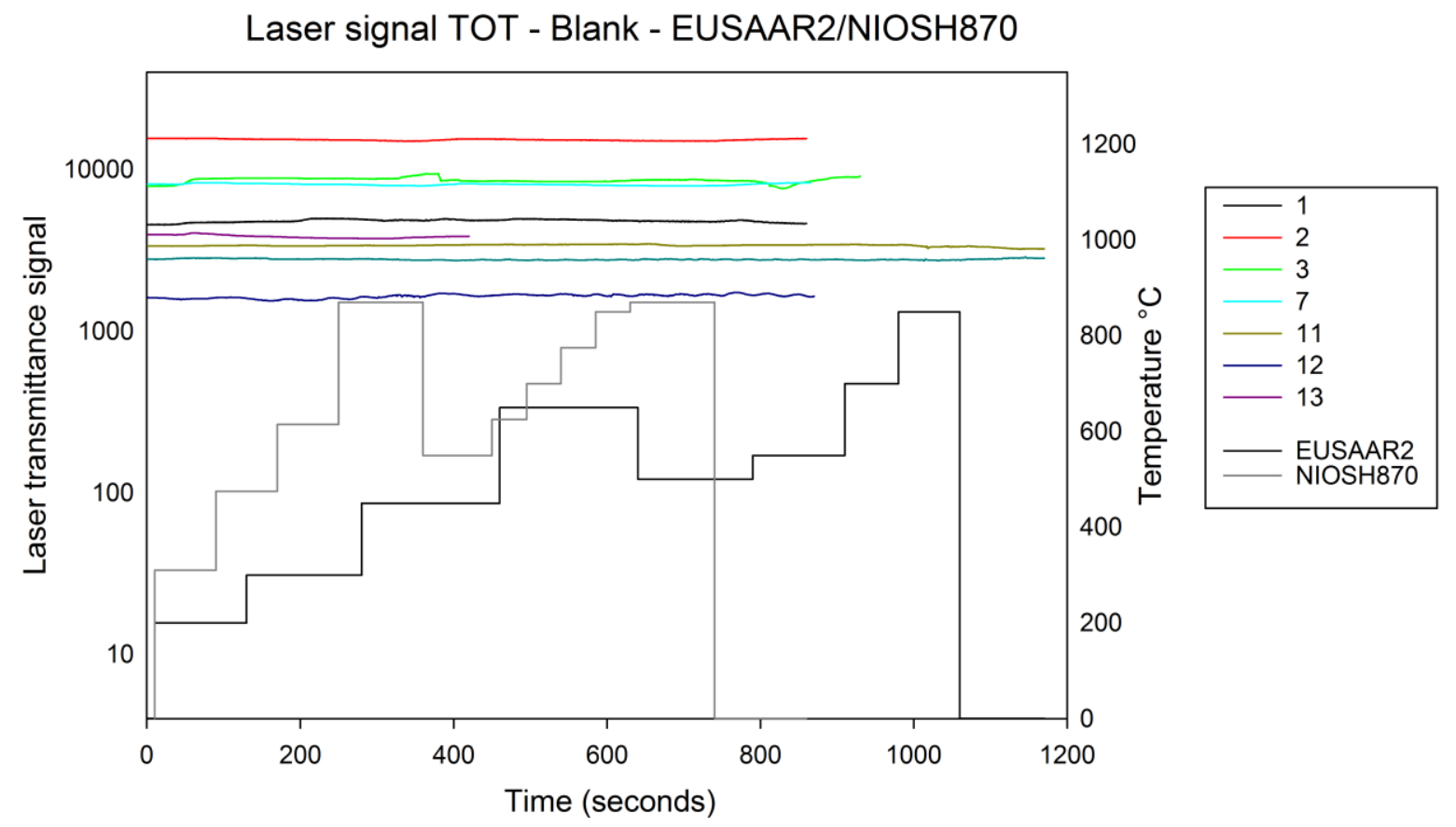

Figure S23. Laser transmittance signal during instrument blank filter analysis with the use of the EUSAAR2 or NIOSH870 thermal protocol. (Participant 13 used a shorter protocol for the specific analysis). 


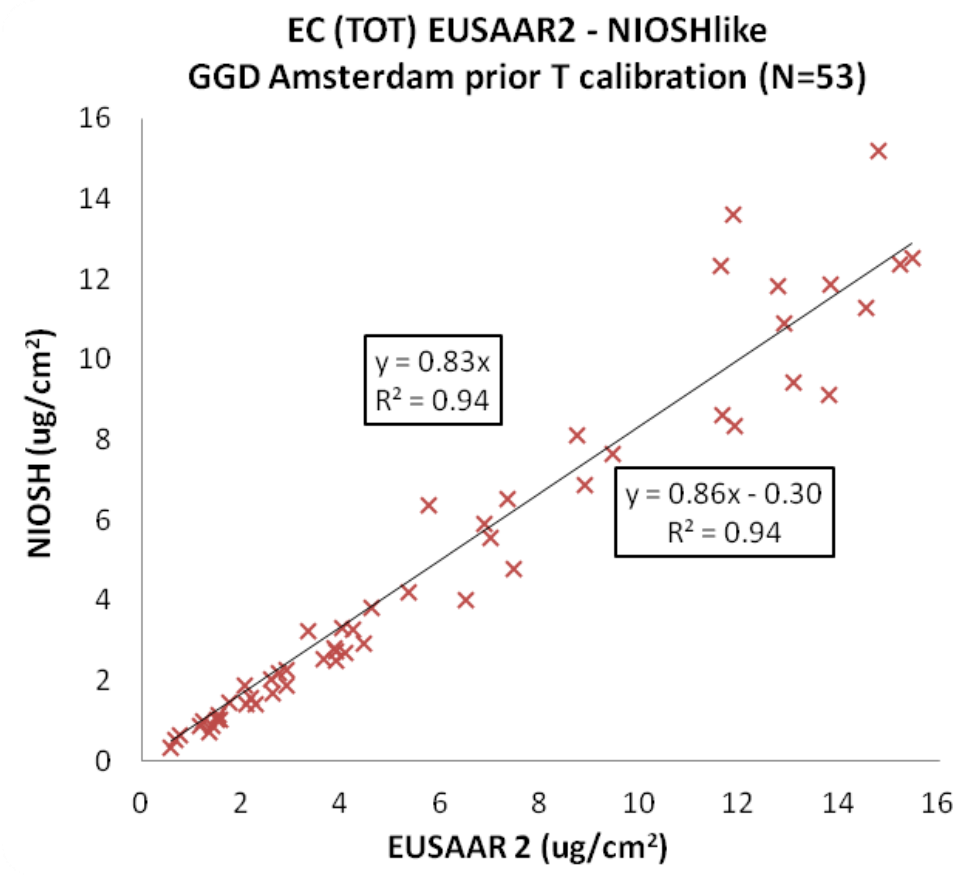

Figure S24. Comparison of EC (TOT) results between EUSAAR2 and NIOSH870like protocols. Analysis performed by GGD Amsterdam prior to temperature calibration (Panteliadis, 2011; 2012; Keuken, et al., 2013).

\section{References}

Keuken, M. P., Jonkers, S., Moerman, M., Jedynska, A. D., Verbeek, R., Visschedijk, A, van den Elshout, S., Panteliadis P. and Velders, G. J. M. (2013). Modelling elemental carbon at regional, urban and traffic locations in Netherlands Atmos. Environ., 73, 73-80.

Panteliadis, P. (2011). Elemental and Organic Carbon measurements RIVM location 644 Cabauw, 2010. GGD/LO 11-1125. Public Health Service Amsterdam, Department of Air Quality.

Panteliadis, P. (2012). Elemental and organic carbon measurements RIVM location 644 Cabauw. GGD/LO 12-1143. Public Health Service Amsterdam, Department of Air Quality. 